\title{
Conceptual and perceptual memory: Retrieval orientations reflected in event-related potentials
}

\author{
Georg Stenberg ${ }^{\mathrm{a}, *}$, Mikael Johansson ${ }^{\mathrm{b}}$, Ingmar Rosén ${ }^{\mathrm{b}}$ \\ a Department of Psychology, School of Behavioural Sciences, Kristianstad University, \\ SE-291 88 Kristianstad, Sweden \\ ${ }^{\mathrm{b}}$ Departments of Psychology and Clinical Neuroscience, Lund University, SE-221 00 Lund, Sweden
}

Received 6 January 2005; received in revised form 29 October 2005; accepted 7 November 2005

Available online 9 January 2006

\begin{abstract}
In memory retrieval, search can be guided by mental sets towards different subsets of the available evidence. Such retrieval orientations have been suggested to leave an imprint on event-related potentials (ERPs). The present study aimed at characterizing orientations towards perceptual and conceptual evidence in a recognition task, where pictures and words were studied. In the recognition test, items were presented in either the same format as at study or in the opposite format. A between-subjects manipulation modified the task, instructing an Exclusion group to endorse only items that preserved their format from study, and an Inclusion group to endorse both formats of a studied item. It was hypothesized that exclusion instructions would instil a perceptual and inclusion instructions a conceptual orientation. As a corollary, instructions were expected to dissociate the high end from the low end of the picture-word mirror effect. This expectation was confirmed in a behavioural experiment. In an ERP experiment, retrieval orientations were examined in their effects on correct rejections of new pictures and words. Confirming earlier findings [Hornberger, M., Morcom, A. M., \& Rugg, M. D. (2004). Neural correlates of retrieval orientation: effects of study-test similarity. Journal of Cognitive Neuroscience, 16(7), 1196-1210], a perceptual orientation was accompanied by more positive-going amplitudes over widespread areas. The difference was larger for pictures than for words, supporting behavioural evidence that new pictures are more easily rejected on perceptual grounds than are new words. The Exclusion group showed no ERP evidence of cross-format oldnew effects, despite reaction times indicative of involuntary conceptual recognition. The results indicate that perceptual and conceptual retrieval orientations imprint distinct signatures on ERPs. They
\end{abstract}

\footnotetext{
* Corresponding author. Tel.: +46 44203288.

E-mail addresses: georg.stenberg@bet.hkr.se, georg.stenberg@home.sol.se (G. Stenberg).
} 
further suggest that the examined old-new effects in ERPs are mainly linked to voluntary aspects of memory, even in a task where involuntary memory exerts effects on reaction times.

(C) 2005 Elsevier B.V. All rights reserved.

PsycINFO classification: 2530

Keywords: Object recognition; Explicit memory; Pictorial stimuli; Cortical evoked potentials; Electrophysiology

\section{Introduction}

Memory is used in a variety of ways, and recent years have seen a proliferation of proposed memory systems to accommodate them (Schacter, Wagner, \& Buckner, 2000). In spite of this diversity, theoretical models of memory have been remarkably successful in mimicking the typical findings within the confines of a unitary store and a single measure of memory strength (Clark \& Gronlund, 1996; Ratcliff \& McKoon, 2000). Some findings have, however, raised stumbling blocks for unitary memory theories, among them the mirror effect (Reder, Angstadt, Gary, Erickson, \& Ayers, 2002). The present study examines uses of memory in which interest is geared towards either perceptual of conceptual features of the sought-for memory images. It aims to test the proposition that these two orientations are characterised by separate signatures in brain event-related potentials (Hornberger, Morcom, \& Rugg, 2004). It also tests the related proposition that a perceptual orientation preserves the picture-word mirror effect, whereas a conceptual orientation eliminates it.

\section{Retrieval orientations}

An orientation of retrieval attempts toward a certain type of material can benefit retrieval, particularly if the stance adopted at test matches the one used at encoding. Many empirical observations support the suggestion that recognition memory benefits from an overlap between processing during encoding and processing during retrieval (Weldon \& Roediger, 1987). In particular, the distinction between perceptual and conceptual processing has proved important in this regard. If processing at study is conceptual rather than perceptual, performance in a conceptual memory test, such as recall or recognition, will improve. Conversely, perceptual encoding will enhance performance in tasks that repeat the same perceptual processes at test (Weldon, Roediger, Beitel, \& Johnston, 1995).

Event-related potentials (ERPs) have been used to study brain responses during memory processing with a high time resolution. The kind of processing that retrieval cues are subjected to can best be observed in correct rejection trials of new items, without confounding influences from retrieved material, as would be the case with old items. The specific form of processing that is applied to retrieval cues has been called retrieval orientation (Rugg \& Wilding, 2000). It has been the subject of a few recent ERP studies. For example, frontal responses, particularly on the left side, have been found to differentiate a more specific from a more general memory search in the 600-800 ms range (Ranganath \& Paller, 2000). Deep versus shallow processings in the study phase have been found to engender different ERP patterns during retrieval processing (Rugg, Allan, \& Birch, 2000).

Robb and Rugg (2002) had participants study lists of pictures or words, in different blocks, and used words as recognition cues for both. The processing of new words differed 
markedly depending on whether it occurred in the context of studied words or studied pictures. In the latter case, where there was no perceptual overlap between study and test, responses were more negative, from about $250 \mathrm{~ms}$, over widespread areas. Difficulty was also manipulated but proved unrelated to the retrieval orientation effect.

Herron and Rugg (2003a) used mixed lists of pictures and words for study, and word lists for test. In one test block, positive response was to be given only to words corresponding to the studied pictures, and the studied words were to be rejected along with new words. In another test block, positive responses were given only to the studied words. Responses to new words varied between blocks as a result of the different retrieval orientations. As found by Robb and Rugg, responses to items that repeated the studied format were more positive, with an onset of about $300 \mathrm{~ms}$ and a long duration, as well as a widespread distribution.

In a further investigation (Hornberger et al., 2004), study and test formats (picture/ word) were factorially varied. Again, responses to new items were more positive in the condition where there was greater perceptual overlap between study and test (word-word or picture-picture, compared to crossovers). A follow-up experiment showed that the perceptual overlap need not be complete, as long as it is greater than the alternative, i.e. visual word after the study of auditory word gave more positive amplitudes than visual word after picture. The effect set in around $300 \mathrm{~ms}$ and had not dissipated at the end of the epoch. Topographical distribution of the effect was again diffuse, with a central maximum. There was, however, a more anterior distribution of the effect for new words than for new pictures. The authors examined a number of potential explanations of the retrieval orientation effect, among them differing familiarity of the cues in the sense of global-matching models (Clark \& Gronlund, 1996). The account favoured by the authors is, however, one that derives from the degree of conceptual processing: " ... it is proposed that the more that a cue representation must be constrained to the semantic/conceptual level, the more negative-going will be the associated ERPs" (Hornberger et al., 2004, p. 1205).

In the present study, retrieval orientation effects were investigated along the lines proposed by Hornberger et al. Specifically, we aimed to induce retrieval orientations that varied in the weight they gave to perceptual and conceptual features. We expected this shift in emphasis to affect ERPs to new test stimuli by producing more negative-going amplitudes accompanying a conceptual orientation, and more positive-going amplitudes with a perceptual orientation.

\section{The tasks}

Our participants were assigned to two conditions: the Format Exclusion task and the Format Inclusion task. In both conditions, mixed lists of pictures and words were presented for study. In the test, the Exclusion group was instructed to endorse only stimuli that appeared in the same format as when studied. The Inclusion group was instructed to endorse an item in both the original and the opposite format; e.g. a studied picture was to be recognized both as a picture and as a word (see Table 1).

The Inclusion task induces greater reliance on conceptual than perceptual cues, because studied items are to be recognised across formats. Perceptual features are valid cues only if they are recognized as studied, and no conclusion can be drawn from unfamiliar perceptual features. In contrast, in the Exclusion task, unfamiliar perceptual features deliver valuable information; they blaze a trail for a quick "new" judgement. Recognition can be based 
Table 1

The stimulus categories in the experiments

\begin{tabular}{lll}
\hline & Test format & \\
\cline { 2 - 3 } & Picture & Word \\
\hline Studied format & $\mathrm{PP}^{\mathrm{a}, \mathrm{b}}$ & $\mathrm{PW}^{\mathrm{a}}$ \\
Picture & $\mathrm{WP}^{\mathrm{a}}$ & $\mathrm{WW}^{\mathrm{a}, \mathrm{b}}$ \\
Word & $\mathrm{NP}$ & $\mathrm{NW}$ \\
New & & \\
\hline
\end{tabular}

a To be endorsed as old by the Inclusion group.

b To be endorsed as old by the Exclusion group.

entirely on perceptual appearance. Conceptual cues can be disregarded, because they are attended to only at the peril of triggering false alarms to cross-format items.

Whereas we will eventually examine the effects of perceptual and conceptual orientations on event-related potentials, a behavioural experiment will first be performed. It assesses the influence of the two orientations on recognition accuracy, more specifically the extent to which accuracy conforms to the mirror effect.

\section{The mirror effect}

The mirror effect is an often noted regularity of recognition memory: wherever two classes of stimuli differ in their memorability, the more memorable class tends to have both a higher hit rate and a lower false alarm rate (Glanzer \& Adams, 1985). In other words, the type of stimulus that is more easily recognised as old, when old, is also more easily rejected as new, when new. Typical examples are low-frequency words versus high-frequency words, and pictures versus words. The mirror effect is nearly ubiquitous in recognition memory, yet it poses a problem for many models of memory.

Most quantitative models of memory are of the global-matching type (reviewed by Clark \& Gronlund, 1996). Recognition is characterized as the process of matching a test probe against all traces in memory, and the resulting quantity, a measure of memory strength or familiarity, is compared to a criterion, leading to a recognition decision. The problem with the mirror effect arises out of this. If two types of stimuli, such as pictures and words, differ on a single dimension of overall memory strength, then it is difficult to explain why pictures are stronger items than words when studied, but less strong than words when unstudied.

Some theorists have chosen to abandon the idea of a single dimension underlying recognition decisions. Instead they have posited two different processes, one that creates the hitrate part, and another that creates the false-alarm part. In particular, recollection is often assumed to be the main contributor to the hit-rate part, while familiarity causes the falsealarm part (Joordens \& Hockley, 2000; Reder et al., 2000). Efforts to prove this proposition have centred on manipulations that alter the hit rate, e.g. by making recollection more difficult, while leaving the false-alarm part unaffected. For example, Joordens and Hockley (2000) presented words for incidental study in a long lexical decision task. In the ensuing recognition test, accuracy was low, because of the difficulty, and importantly, hit rates did not differentiate between high- and low-frequency words. False alarm rates were greater for high-frequency words, as is commonly found. The authors interpreted this as reflecting a familiarity advantage for common words, unaffected by the difficulty manipulation that harmed recollection. 
Other theorists have chosen to preserve the unitary memory model, encapsulated in a single measure of memory strength. Importantly, if this strength measure is remapped to a likelihood ratio scale, findings such as the mirror effect can be accommodated within the model. This solution, originally proposed by Glanzer and co-workers (Glanzer, Adams, Iverson, \& Kim, 1993), has been adopted in other recent memory models, such as REM (Shiffrin \& Steyvers, 1997).

The most widely studied mirror effect is the one involving word frequency, but other pairs of stimulus classes exhibit the same phenomenon, such as concrete versus abstract words, and pictures versus words. In their original article on the mirror effect, Glanzer and Adams (1985) reviewed 11 studies contrasting memory for pictures with memory for words, all but 2 of which exhibited both higher hit rates and lower false alarm rates for pictures. However, some later studies have found that the mirror effect does not always apply (Mintzer \& Snodgrass, 1999; Stenberg, Radeborg, \& Hedman, 1995). These have been studied where an element of format change between study and test has been included, i.e. where studied pictures could be tested as (the corresponding) words, and vice versa. Stenberg et al. (1995) had participants study pictures and words, and tested both formats of each studied item, instructing participants to endorse both formats if one of them had been studied. In three experiments, they found this procedure to result in higher false alarm rates (and slower reaction times) for new pictures than for new words, the opposite of the normal finding. Hypothesizing that a slower, conceptual memory search was responsible for this pattern, they performed a fourth experiment, in which both formats were presented together at study, but singly at test. Because the study had made both formats familiar, recognition decisions could be made on the basis of a perceptual match, and the normal mirror effect was expected, and found.

Mintzer and Snodgrass (1999) replicated and extended these findings of a reversed picture-word mirror effect. After a picture study block, new pictures were rejected more accurately than new words. After a word study block, new pictures, now suspected of perhaps being a studied word in disguise, attracted more false alarms than new words. Thus, whether a mirror effect was observed or not depended on participants' expectations about a match between the study format and the test format.

In summary, recognition of studied pictures is reliably more accurate than recognition of studied words. The rejection of new pictures is usually more accurate than the rejection of new words, but certain conditions eliminate this advantage. The possibility that the mirror effect can be abolished is of theoretical interest, because such a dissociation can bolster the case for two-process theories in the ongoing debate about the nature of recognition memory (Arndt \& Reder, 2002; Joordens \& Hockley, 2000; Reder et al., 2002; Reder et al., 2000). In this study, the mirror effect was used as a probe for perceptual and conceptual orientations, following suggestions from earlier work (Mintzer \& Snodgrass, 1999; Stenberg et al., 1995) that participants' orientation toward a conceptual or perceptual match can switch the effect on or off.

\section{Purpose}

A behavioural experiment was first performed to examine the effectiveness of the Inclusion-Exclusion task manipulations. In particular, we were interested in dissociating the high and the low ends of the mirror effect (i.e. the hit rate and the false-alarm parts). We expected to find the normal mirror effect in the Exclusion task, which geared participants toward perceptual features, enabling them to reject the unfamiliar. Novelty rejection can 
be performed more efficiently with pictures than with words, because of their greater perceptual distinctiveness, hence picture superiority is expected to prevail at the low end as well as the high end.

In the Inclusion task, on the other hand, novel perceptual features are not diagnostic, because they may conceal a conceptually (cross-format) studied item. Hence, we expected the rejection of new items to be more difficult, and we expected new pictures to lose their edge over new words in the Inclusion condition. If this manipulation of the mirror effect proved successful, we would interpret it as indicating that instructions had indeed induced a perceptual and a conceptual retrieval orientation.

\section{Experiment 1}

\subsection{Method}

\subsubsection{Participants}

A total of 40 students at Växjö University participated and received a cafeteria voucher in compensation. Half were randomly assigned to the Exclusion and half to the Inclusion condition. Mean age was 25 (range: 19-39) and gender distribution was 8/11 (male/female) in the Exclusion group. For the Inclusion group the corresponding figures were 29 years (19-47) and 7/13. (Data were missing on the age and sex of one participant.)

\subsubsection{Materials}

A set of 322 colour photographs were selected from a commercially available collection (Hemera Photo Objects 5000; Hemera Technologies, Inc.) to be used as stimuli, along with the corresponding Swedish names. Selection criteria were ease of naming and familiarity. The photographs represented common objects, such as car, bus, cheese or pizza. Each object was displayed separately against a uniform white background.

For each participant, a random subset of the stimulus material, consisting of 192 items, was selected and further subdivided into four blocks. Of the 48 items in a block, 32 were designated to be studied, half as pictures and half as words. In the test phase, studied items reappeared, half in the same format (picture/word), and half in the opposite format. The studied items were mixed with new items, half pictures and half words. Thus, in each block, 16 pictures and 16 words were studied. In the test phase, eight studied pictures reappeared as pictures (type PP), eight studied pictures appeared as words (PW), eight studied words appeared as pictures (WP), and eight studied words reappeared as words (WW). Eight new items were presented as pictures (NP), and eight as words (NW). Summed over the four blocks, there were therefore 32 items of each of six test types. No item was used in more than one block. All assignments were randomized anew for each participant.

\subsubsection{Procedure}

Participants were tested in small groups of 2-5, each seated in an individual booth with a computer. After being given instructions and some examples, participants ran the experiment program at an individual pace. Instructions were identical for the two groups, except for the crucial part concerning responses to items in a changed format. It was explained by examples, for example, a picture of an ambulance could appear in the study phase, to be followed by the word "ambulance" in the test. Similarly, the word "doll" could be studied, and a picture of a doll could thereafter appear in the test. For items such as these, the 
Exclusion group was instructed to respond "New", whereas the Inclusion group was instructed to respond "Old". Speed of responding was not mentioned in the instructions.

Each trial in the study phase displayed a picture or a word for $2 \mathrm{~s}$, with no overt response required. Instructions were to watch the stimuli and try to remember them. In the test phase, each item was shown until a response was given, or until $5 \mathrm{~s}$ had elapsed, whichever happened first.

Feedback concerning the correctness of the response and the cumulative percentage correct was given after each item and displayed for $1.5 \mathrm{~s}$. In all, four study-test cycles, each with 32 study items and 48 test items were completed. The test phase followed immediately after the last study item.

\subsection{Results}

The proportions of correct responses and the reaction times for all test categories are shown in Table 2. The data of primary interest are the responses to the PP and WW categories, which form the Hit-Rate portion of the mirror effect, and to the NP and NW categories, which form the false-alarm part.

\subsubsection{Same-format items}

6.2.1.1. Accuracy. The proportions of correct responses for the PP and WW categories were tested in a $2 \times 2$ Anova, using Format (Picture/Word) and Group (Inclusion/Exclusion) as factors. There were no differences between groups (main effect and interaction, both $F<1.3$ ), but there was a reliable effect of Format, because pictures were better recognised than words, $F(1,38)=126.46 ; p<0.001$. Thus, the hit-rate portion of the mirror effect was preserved in both instruction conditions.

6.2.1.2. Reaction time. A further analysis of reaction times in Hit trials for both groups involved the same two factors as the accuracy analysis. The main effect of Format was significant, $F(1,38)=107.45 ; p<0.001$, as was the main effect of Group, $F(1,38)=18.68$; $p<0.001$. There was no interaction $(p=0.13)$. The generally slower "yes"-responses from the Exclusion participants could reflect a bias to respond "no", as a result of the greater proportion of to-be-rejected items in this condition.

\subsubsection{New items}

False alarms were lower for pictures (4\%) than for words $(12 \%)$ in the Exclusion group, but the reverse was true in the Inclusion group: $20 \%$ for pictures versus $12 \%$ for words. Thus, the false alarm portion of the mirror effect was preserved in the Exclusion group

Table 2

Accuracy and reaction times in Experiment 1

\begin{tabular}{lllllll}
\hline & PP & WW & NP & NW & PW & WP \\
\hline $\begin{array}{l}\text { Accuracy } \\
\text { INC }\end{array}$ & 0.983 & 0.750 & 0.802 & 0.878 & 0.899 & 0.783 \\
EXC & 0.943 & 0.733 & 0.976 & 0.877 & 0.852 & 0.976 \\
RT & & & & & & 1027 \\
INC & 754 & 998 & 1350 & 1254 & 1085 \\
EXC & 893 & 1225 & 995 & 1182 & 1325 & 1059 \\
\hline
\end{tabular}


$[t(19)=5.77 ; p<0.001]$, but it was reversed in the Inclusion group $[t(19)=-3.69$; $p=0.002]$.

A $2 \times 2$ ANOVA of false alarms showed an interaction, Group $\times$ Format: $F(1,38)=42.56 ; p<0.001$, as well as a main effect of Group: $F[1,38]=11.06 ; p=0.002$. There was no difference between groups for words, $t(38)=0.03 ; p=0.98$ but picture lures were rejected very consistently by the Exclusion group, and much less so by the Inclusion group, $t(38)=6.49 ; p<0.001$.

\subsubsection{Cross-format items}

6.2.3.1. Inclusion. The Inclusion group showed better recognition for cross-format items studied as pictures (PW) than items studied as words (WP), $t(19)=6.08 ; p<0.001$. An Anova performed on all four types of to-be-endorsed items (PP, WW, PW, and WP) showed that perceptual similarity (same-format versus cross-format) had no main effect, $F(1,19)=1.49$, N.S. This confirms that, on the whole, participants could perform the task as intended, with few misses due to format changes. There was, however, an interaction, $F(1,19)=8.99 ; p=0.007$, due to a slight decrement when the studied pictures changed format (PW), but no such decrement, indeed an improvement, for studied words.

6.2.3.2. Exclusion. The Exclusion group made more correct responses when rejecting WP foils than when rejecting PW foils, $t(19)=4.36$; $p<0.001$. Thus, as was the case for completely new items, unseen pictures were rejected more accurately than unseen words, in keeping with the mirror effect.

As a manipulation check, an Anova was performed on all to-be-rejected items (NP, NW, WP, PW). It confirmed that participants could reject items without making false alarms due to conceptual acquaintance, because there was no main effect or interaction involving Conceptual Study (PW and WP versus NW and NP), both $F(1,19)<1.38$; N.S.

There was, however, a cost in reaction time, because participants were slower to reject conceptually studied, cross-format items than completely new items. A $2 \times 2$ Anova on RTs revealed an effect of conceptual study, $F(1,19)=55.49 ; p<0.001$.

\subsection{Discussion}

The experiment showed that instructions can selectively eliminate the false-alarm part of the picture-word mirror effect while preserving the hit-rate part. It seems that a mechanism serves to reject novel stimuli off-hand, if the perceptual format can be trusted, as was the case in the Exclusion condition. Importantly, the novelty rejection mechanism was used mainly with pictures, suggesting that the perceptual format of words was not trusted to be memorable enough to serve as diagnostic. The Inclusion condition threw the novelty rejection mechanism out of kilter, because low perceptual familiarity could no longer be trusted as a diagnostic. This inflated the false alarm rate for pictures, but left false alarms for words unaffected.

The fact that Inclusion instructions selectively affected the false alarm rate for pictures, not for words, excludes the alternative explanation that Inclusion participants noticed that there were more than $50 \%$ targets in the test, and simply adjusted their criteria in a liberal direction across the board.

The finding that attention to perceptual format, as induced by instructions, preserves the mirror effect, while attention to conceptual features disables it, suggests that the 
affected lower end of the mirror effect is operated by a perceptual rejection mechanism. This conjecture will be submitted to an electrophysiological test. We expect a perceptual retrieval orientation to reveal itself in increased positive amplitudes in the relevant perceptual (visual) areas, accompanying the scrutiny of stimuli for novel, unfamiliar features, which can expose the stimulus as a foil.

At the other extreme, we expect a conceptual retrieval orientation to reveal itself in brain responses to cross-format items. It is an open question whether these responses are guided by a voluntary, recollection-like mechanism. If so, old-new-differences in ERPs will show up only in the Inclusion group. If, on the other hand, conceptual retrieval operates as an automatic, priming- or familiarity-like mechanism, it will be manifest in both groups, revealed by "old"-indicative ERPs to the cross-format categories WP and PW. The behavioural evidence gathered so far gives us little guidance as to which alternative will prevail, for the accuracy of the responses indicated a controlled process, but the reaction times suggested a possibly automatic mechanism that recognised cross-format items and interfered with negative responses in the Exclusion task.

\section{Experiment 2}

The second experiment was conducted with the same Inclusion/Exclusion tasks and the same stimulus material. Because it involves recognition memory as reflected in ERPs, memory effects will be briefly reviewed.

\subsection{Old-new effects}

A number of studies have investigated the effects of memory on ERPs, operationalized as old-new differences. Current interest in the area has implicated the distinction between recollection and familiarity, which has attracted much attention in recent years (Yonelinas, 2002). Familiarity is mostly thought of as a graded, signal-detection-like measure of memory strength, whereas recollection is a thresholded process, bringing context and associative detail to mind. In early dual-process theories of recognition, familiarity was often thought to depend on perceptual processing, and recollection was seen as relying on conceptual processes (Mandler, 1980). However, this parallelism is not perfect, because familiarity can be based on conceptual fluency (Wagner \& Gabrieli, 1998), and recollection can recapitulate perceptual processing. Therefore, later theories have come to see the two distinctions as relatively independent (Yonelinas, 2002).

Research with event-related potentials has suggested that different, separable brain responses are associated with recollection and familiarity (Mecklinger, 2000; Rugg \& Allan, 2000; Rugg \& Yonelinas, 2003). Old items elicit an early (300-500 ms), attenuated midfrontal negativity, sometimes called the FN400, when they evoke a sense of familiarity. A later (400-900) positive component, maximal over left parietal areas, is evoked by items that are recollected, i.e. remembered with qualitative details. The two components have been dissociated, by using foils similar to studied items, e.g. the plural form of a word studied in the singular (Curran, 2000), or a mirror-reversed form of a picture (Curran \& Cleary, 2003). Such similar items evoke the familiarity component, but yield only a diminished recollection component. Other evidence has indicated that similarity need not be narrowly perceptual to produce the FN400, because visual word presentation after auditory word study can produce the effect (Curran \& Dien, 2003), and so can semantic associates of 
studied words (Nessler, Mecklinger, \& Penney, 2001). A suggested locus of the effect is the anterior part of the medial temporal lobe (Aggleton \& Brown, 1999; Brown \& Aggleton, 2001).

The two-process account has linked the parietal component to recollection, because it is sensitive to variables such as deep versus shallow encoding (Paller \& Kutas, 1992), and full versus divided attention (Curran, 2004). It is thought to arise from interactions between hippocampus and the cortex (Rugg \& Allan, 2000), and normally accompanies only successful retrieval, i.e. hits in signal-detection terms, not misses, and usually not false alarms (but see Düzel, Yonelinas, Mangun, Heinze, \& Tulving, 1997). There have, however, been dissenting opinions concerning the two-process account (Finnigan, Humphreys, Dennis, \& Geffen, 2002; Yovel \& Paller, 2004), which have questioned the dissociations of parietal and early frontal effects or their associations with recollection and familiarity.

Lastly, a late component, from about $600 \mathrm{~ms}$ and often until the end of the recording epoch, is sometimes observed over right frontal areas in tasks where the participant critically scrutinizes the products of retrieval before or after delivering the response. Such may be the case in source memory tasks (Senkfor \& Van Petten, 1998), in recall (Allan \& Rugg, 1997) and in associative recognition (Van Petten, Luka, Rubin, \& Ryan, 2002).

\subsection{Picture and word recognition in ERPs}

Although words are the typical material in ERP memory studies, pictures have shown similar memory effects, including the separation of components linked to recollection and familiarity (Curran \& Cleary, 2003). Memory for pictures and words have also been studied with special attention to format changes between conceptual equivalents (Kazmerski \& Friedman, 1997; Schloerscheidt \& Rugg, 2004). Kazmerski and Friedman had participants perform a semantic orienting task on a mixed list of pictures and words. At test, one half of the items reappeared in the same format, while the other half was replaced by the opposite format. Test items presented in the same format evoked memory effects in both a direct and an indirect memory task, but items in a changed format only in the direct task, and even so, only for one category of format change (word to picture). Thus, memory effects appeared to be perceptually specific to some degree.

Schloerscheidt and Rugg (2004) used a similar format-change paradigm within a direct recognition task. Test format was manipulated as a between-participants factor, i.e. one group saw only test pictures and another only test words. In both cases, test stimuli corresponded to either items studied in the same format, items studied in the other format, or were completely new. With this task, reliable cross-format as well as within-format old-new effects were obtained. The early frontal, putative familiarity effect was, however, restricted to within-format repetitions. The parietal component, linked to recollection, accompanied both across-format and within-format items, although with somewhat different latencies and amplitude. In general, old-new effects were smaller and later if the format had changed.

The study by Herron and Rugg (2003a), mentioned earlier, made the observation that when studied pictures were the targets, studied words, despite being rejected, elicited parietal old-new effects. When words were the targets, studied pictures did not produce the same effect, i.e. only when there was perceptual overlap between study and test, was there a recollection effect for excluded stimuli (all the test stimuli were words). In a different study by the same authors (Herron \& Rugg, 2003b), exclusion instructions were given as in Jacoby's Process Dissociation Procedure (Jacoby, 1991) in two experiments. Depending on 
strategies induced by the study tasks, excluded stimuli elicited ERP recollection effects in one experiment, but not in the other. The determining factor was the ease with which to-be-excluded stimuli could be distinguished from to-be-included. Thus, exclusion instructions can have a variable relation to ERP old/new effects. To-be-excluded items sometimes produce recollection effects in ERPs, sometimes not. The present study used an exclusion, and an inclusion task, and examined, among other things, whether there were any old-new effects for to-be-excluded stimuli.

\subsection{Purpose}

The experiment was essentially a replication of Experiment 1, although adapted to the need for many trials of each type, to form stable ERP averages. Therefore, the experiment was lengthened to 300 test trials (and 200 study trials). To keep performance high, the session was divided into 10 study-test cycles.

Three questions were in focus:

1. Do perceptual and conceptual retrieval orientations produce the ERP effects predicted by Hornberger et al. (2004), i.e. more negative-going amplitudes accompanying a conceptual orientation?

2. Do to-be-excluded stimuli produce involuntary old-new effect in ERPs (cf. Herron \& Rugg, 2003a, 2003b)? Some memory effects - such as recollection - are typically voluntary, whereas others - such as priming - appear to be beyond conscious control. A few studies have been able to identify ERP concomitants of perceptual priming (reviewed by Paller, 2004), and more rarely, of conceptual priming (Olichney et al., 2000). Our interest focused here on responses to changed-format items in the Exclusion group, and possible signs of conceptual memory effects therein.

3. Do differences in the to-be-retrieved material determine the scalp distribution of oldnew effects, suggesting the operation of different neuronal populations? Most studies of ERP old-new effects have found relatively invariant distributions, but a few have sought for, and found, material dependence in the localization of effects (Mecklinger, 2000; Rösler, Heil, \& Hennighausen, 1995). Our design allows us to contrast same-format versus changed-format items, as well as studied pictures versus studied words, independently of test format.

\subsection{Method}

\subsubsection{Participants}

Data are reported from 32 participants; 16 in each group. Another three subjects were tested but not analyzed because of incomplete data or excessive artefacts in the physiological data. Gender distribution (female/male) was $8 / 8$ in the Inclusion group, and 10/6 in the Exclusion group. The participants were psychology students at the University of Lund, who were given a cinema ticket for their participation. Mean age was 25 and 24 years, respectively, in the Inclusion and Exclusion groups (ranges: 21-36 and 19-38).

\subsubsection{Stimuli}

The same pool of 322 stimuli as in Experiment 1 was used, from which a random selection of 300 was made for each participant. The selection was further randomly divided into 
10 sets. Of the 30 items in a set, 20 were designated to be studied, half as pictures and half as words. As in Experiment 1, six equiprobable types of stimuli appeared at test: PP, WW, NP, NW, PW and WP. Summed over the 10 blocks, there were 50 items of each of the test types. No item was used in more than one block. All assignments were randomized anew for each participant.

\subsubsection{Procedure}

After application of the electrode cap, instructions were given on-screen, and the experimental session proceeded in 10 study-test cycles, with self-paced pauses in between. The whole recording session, excluding electrode application, took between 35 and $40 \mathrm{~min}$.

Participants used their right hand to respond, by pressing the " 1 " or " 2 " keys on the numerical keyboard. Instructions were the same as in Experiment 1, except that both speed and accuracy were mentioned.

Each trial in the study phase displayed a picture or a word for $2 \mathrm{~s}$, with no overt response required. Instructions were to watch the stimuli and try to remember them. In the test phase, each item was shown for $3.9 \mathrm{~s}$. Feedback concerning the correctness of the response and the cumulative percentage correct was given after each item, in order to keep motivation and performance high and to keep subjects' criteria well calibrated. The feedback was shown for $1.6 \mathrm{~s}$, bringing the total length of each trial to $5.5 \mathrm{~s}$.

\subsubsection{Electrophysiological recording and signal processing}

The EEG was recorded using tin electrodes in an electrode cap (NeuroScan). Electrodes were placed on the positions of the 10-20-system, and referenced to the left mastoid during recording. Additional electrodes were applied to monitor vertical eye movements (VEOG; above and below the left eye), and horizontal eye movements (HEOG; outside the outer canthi). One electrode was applied to the right mastoid and recorded for use in later rereferencing.

Amplifiers were set to accept frequencies from 0.1 to $30 \mathrm{~Hz}$, and digitization was performed at a rate of $250 \mathrm{~Hz}$. The data were saved continuously to disk during the session for later off-line processing. The files were visually inspected, and EEG stretches with large artefacts were rejected. Consistently bad channels were replaced by an interpolation from the closest neighbours. This proved necessary for an average of 0.4 channels per participant (range: 0-2).

From the continuous EEG-files, a template for a typical blink artefact was computed, and corrections for blinks were made to the EEG channels using a regression approach implemented in the NeuroScan software. All EEG channels were re-referenced digitally to an average of the left and the right mastoid. The files were segmented into epochs, consisting of $300 \mathrm{~ms}$ prestimulus and $2000 \mathrm{~ms}$ poststimulus, and the epochs were baseline-corrected by subtraction of the prestimulus average. An artefact rejection algorithm discarded epochs where any EEG channel deviated from baseline by more than $150 \mu \mathrm{V}$. The files were digitally low-pass filtered with a cut-off of $15 \mathrm{~Hz}$ and a roll-off of $48 \mathrm{~dB}$. Behavioural data were used to reject all trials with incorrect responses. Finally, averages were formed for the six test types (PP, PW, WP, WW, NP and NW). The average number of sweeps contributing to an average was 41.8 (SD: 6.6$){ }^{1}$

\footnotetext{
${ }^{1}$ For the different conditions: NP: 44.3; NW: 43.7; PP: 44.9; PW: 42.6; WP: 39.3; WW: 35.8.
} 


\subsubsection{Data processing}

Average amplitudes in three time bands were computed. The time intervals selected were 300-500, 500-700, and 700-900. Computations were done for each electrode, but only a rectangular grid of electrode sites was used, because it covered regions of interest, and because the grid arrangement permitted factorial analyses.

Old-new difference waveforms were computed and used in analyses of memory effects. Because the onset latencies of old-new effects at different locations were of interest, ${ }^{2}$ we calculated onset times for each electrode in a $3 \times 3$ grid (symmetrically surrounding $\mathrm{Cz}$ ). Onset of the old-new effect was defined as the first point in a continuous run of at least 5 points $(20 \mathrm{~ms})$, each of which was positive and at least $1.0 \mu \mathrm{V}$ above baseline. The search was performed in the time range $150-800 \mathrm{~ms}$, and it found points satisfying the criteria in $73 \%$ of the cases ( 422 out of 576 , i.e. 16 subjects $* 4$ conditions $* 9$ electrodes). For purposes of analysis, missing values were replaced by the mean of the variable, using SPSS' missing value facility.

\subsection{Results}

\subsubsection{Behavioural data}

7.5.1.1. Same-format items. Accuracy: The proportions of correct responses were first analyzed for the preserved-format old items, i.e. PP and WW. The data were subjected to a $2 \times 2$ mixed model ANOVA, with one within-subjects factor (Picture/Word), and one between-subjects factor (Inclusion/Exclusion Group). There was no effect involving Group (main effect and interaction, $F<1$ ), but there was a reliable picture superiority effect: $F(1,30)=86.59 ; p<0.001$. Both groups were more accurate in recognising pictures than words. Thus, the hit-rate portion of the mirror effect was preserved (Table 3 ).

Reaction time: As in Experiment 1, Hit reaction times were analyzed in a $2 \times 2$ Anova, with Format and Group as factors. There were reliable main effects of Format, $F(1,30)=343.84 ; p<0.001$, and of Group, $F(1,30)=18.07 ; p<0.001$, as well as an interaction, $F(1,30)=11.63$; $p=0.002$. Overall, the Inclusion group responded faster, the difference being especially large in the slower word (WW) condition.

7.5.1.2. New items. For new items, there was an interaction Group $\times$ Format, $F(1,30)=$ $7.17 ; p=0.01$, and a Group effect, $F(1,30)=5.74 ; p=0.02$. For pictures, the Inclusion group made more false alarms than the Exclusion group, $t(30)=3.47 ; p=0.002$, but for words, there was no difference, $t(30)=0.28 ; p=0.78$.

In separate tests of the false alarm portions of the mirror effect, the Exclusion group showed the expected effect, $t(15)=2.55 ; p=0.022$, in which fewer false alarms were made to pictures $(1 \%)$ than to words $(6 \%)$. The Inclusion group showed the opposite pattern $(9 \%$ for pictures and $6 \%$ for words), although this difference was not significant, $t(15)=1.38$; $p>0.10$. Thus, the mirror effect was preserved in the Exclusion group and eliminated in the Inclusion group.

7.5.1.3. Cross-format items. Inclusion: The Inclusion group showed better recognition for cross-format items studied as pictures (PW) than items studied as words (WP), $t(15)=4.81$;

${ }^{2}$ This analysis was suggested to us by an anonymous reviewer. 
Table 3

Accuracy and reaction times in Experiment 2

\begin{tabular}{|c|c|c|c|c|c|c|}
\hline & PP & WW & NP & NW & PW & WP \\
\hline \multicolumn{7}{|c|}{ Accuracy } \\
\hline INC & 0.975 & 0.762 & 0.909 & 0.939 & 0.914 & 0.715 \\
\hline $\mathrm{EXC}$ & 0.964 & 0.785 & 0.990 & 0.945 & 0.923 & 0.984 \\
\hline \multicolumn{7}{|l|}{ RT } \\
\hline INC & 793 & 1056 & 1549 & 1379 & 1087 & 1228 \\
\hline $\mathrm{EXC}$ & 954 & 1335 & 1050 & 1286 & 1394 & 1137 \\
\hline
\end{tabular}

$p<0.001$. A $2 \times 2$ Anova on all four to-be-accepted categories (PP, WW, PW, WP) showed an effect of Picture Study, $F(1,15)=35.89 ; p<0.001$, and, in contrast to Experiment 1, an effect of Format change, $F(1,15)=9.08 ; p=0.009$.

Exclusion: Accuracy - The Exclusion group made more false alarms when rejecting PW foils $(8 \%)$ than when rejecting WP foils $(2 \%), t(15)=4.08 ; p=0.001$. As found earlier, unseen pictures were rejected more accurately than unseen words, in congruence with the mirror effect. A $2 \times 2$ Anova on accuracy for all four to-be-rejected categories (NP, NW, WP, PW) showed no effect of Conceptual Study, $F(1,15)=2.02$; N.S., but an effect of Picture Format at test, $F(1,15)=9.08 ; p=0.009$, i.e. a result as in Experiment 1 .

RT - Rejection latencies were shorter for WP than for PW items, $t(15)=6.96 ; p<0.001$. In other words, cross-format items were rejected faster, if they were tested as pictures. A further analysis was performed of the cross-format RTs in comparison to RTs for completely new items. Cross-format items were rejected more slowly than wholly unacquainted items, a form of negative conceptual priming in the Exclusion group, $F(1,15)=14.94$; $p=0.002$. This applied to both (test) pictures (WP:1137 versus NP:1050; $t[15]=4.91$; $p<0.001$ ) and (test) words (PW: 1394 versus NW: 1,$286 ; t[15]=2.73 ; p=0.016$ ).

\subsubsection{Electrophysiological results}

The waveforms of the test-phase ERPs are presented in Figs. 1-4. Test pictures, i.e. categories PP, WP and NP are shown in Figs. 1 and 3, for the Exclusion and the Inclusion groups, respectively. Similarly, Figs. 2 and 4 show test words (categories WW, PW and NW) for the two groups.

7.5.2.1. Retrieval orientation: correct rejections. Retrieval orientations are preferably studied in the responses to correctly rejected new items. Task effects on ERPs to new items indicate effects on the manner in which the test probes are used to query memory. Based on recent studies (Hornberger et al., 2004), we expected a perceptual orientation to result in more positive amplitudes over widespread areas, as compared to a conceptual orientation.

Further, because the lower part of the mirror effect was a focus of special interest, we searched for a neural correlate of the finding that new pictures were particularly easy to reject for the Exclusion participants. The search for a novelty rejection mechanism thus translated into an expected interaction involving Task $\times$ Format, where we hypothesized the Exclusion-picture combination to stand out with an especially strong response.

Waveforms for correct rejection trials are shown in Figs. 5 and 6. Data were analyzed from a $3 \times 3$ grid of electrodes, comprising the frontal, central and parietal rows, and three time intervals, i.e. 300-500, 500-700 and 700-900. Mean amplitudes were submitted to a 

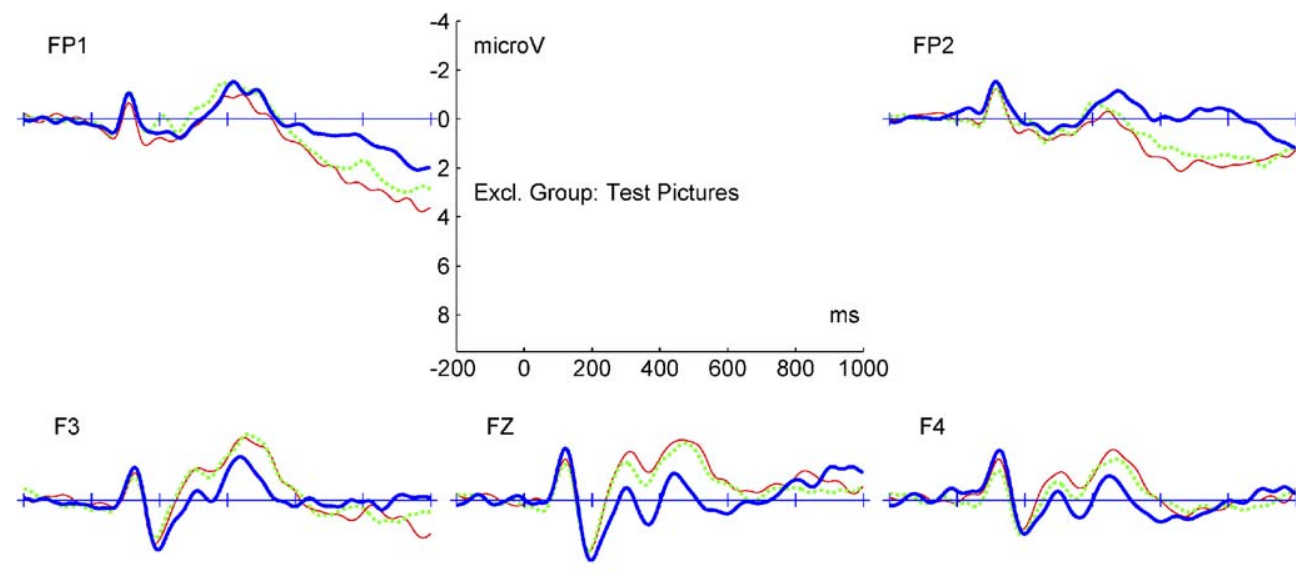

F4

C3

CZ

C4
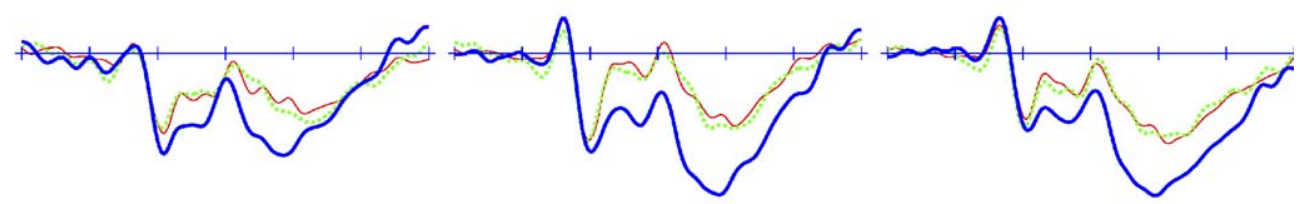

P3

PZ

P4
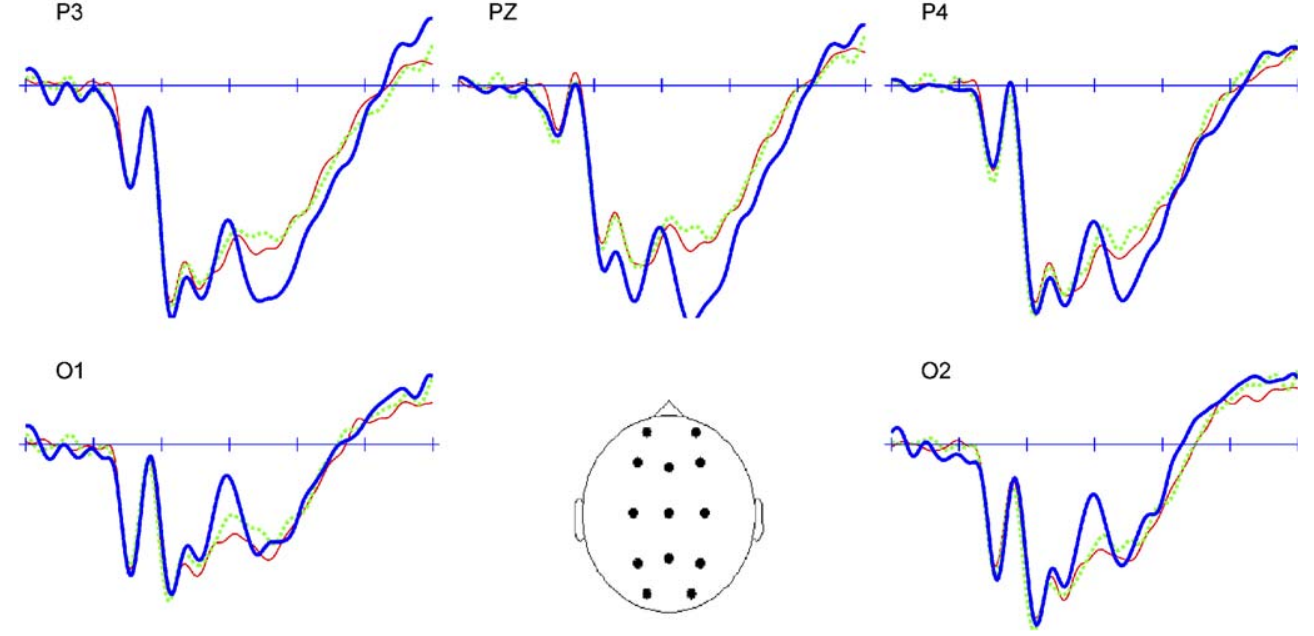

Fig. 1. Waveforms for picture stimuli in the test phase of the Exclusion task. Thin, solid line: new pictures (NP); bold, solid line: old, same-format pictures (PP); dotted line: pictures, studied as words (WP). Inset, schematic view of head showing electrode positions.

Task $(2$ levels $) \times$ Interval $(3$ levels $) \times$ Format $(2$ levels $) \times$ Anterior-Posterior $(3$ levels $) \times$ Lateral ( 3 levels) analysis. The outcome of the Anova is presented in Table 4.

The main effect of Task was reliable because of more positive amplitudes overall in the Exclusion task $(1.27 \mu \mathrm{V})$ than in the Inclusion task $(-0.03 \mu \mathrm{V})$. Further, the three-way interaction Task $\times$ Format $\times$ Anterior/Posterior was significant, and this interaction is illustrated in Fig. 7. The figure suggests larger posterior amplitudes for pictures in the 

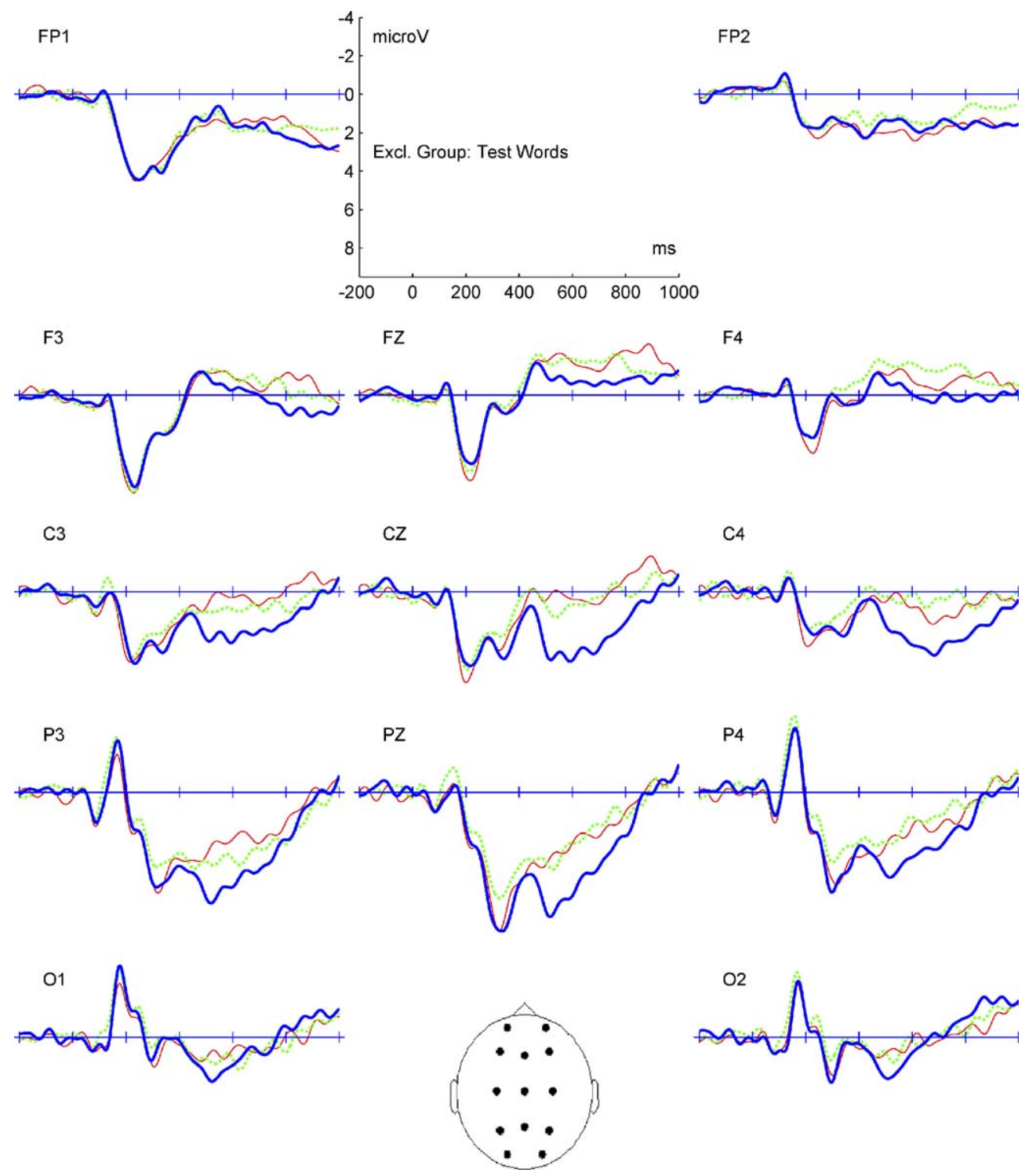

Fig. 2. Waveforms for word stimuli in the test phase of the Exclusion task. Thin, solid line: new words (NW); bold, solid line: old, same-format words (WW); dotted line: words, studied as pictures (PW).

Exclusion task than in the other three Task-Format combinations. To parse the interaction, a separate analysis was performed for the three parietal electrode sites, using Task, Format, and Lateral position as factors. It resulted in a main effect of Task, $F(1,30)=4.54$; $p=0.041$, and a Task $\times$ Format interaction, $F(1,30)=4.78 ; p=0.037$, indicating larger amplitudes in Exclusion-Picture $(4.28 \mu \mathrm{V})$ than in the other cases $(2.58 \mu \mathrm{V}, 1.35 \mu \mathrm{V}$ and $1.34 \mu \mathrm{V}$, in the Exclusion-Word, Inclusion-Picture and Inclusion-Word conditions, respectively). Corresponding analyses for the central and frontal electrodes did not show the same effects $[F \mathrm{~s}(1,30) \leqslant 2.49]$. 



F4

C3

$\mathrm{CZ}$
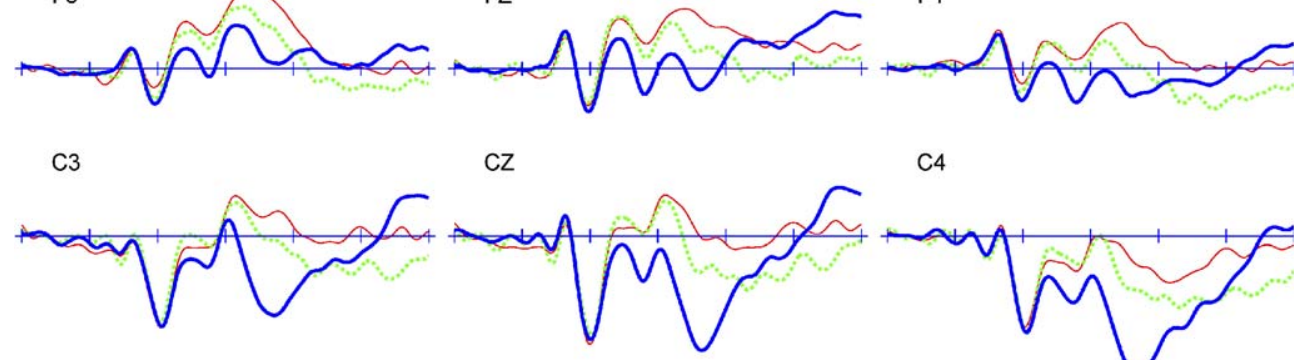

C4
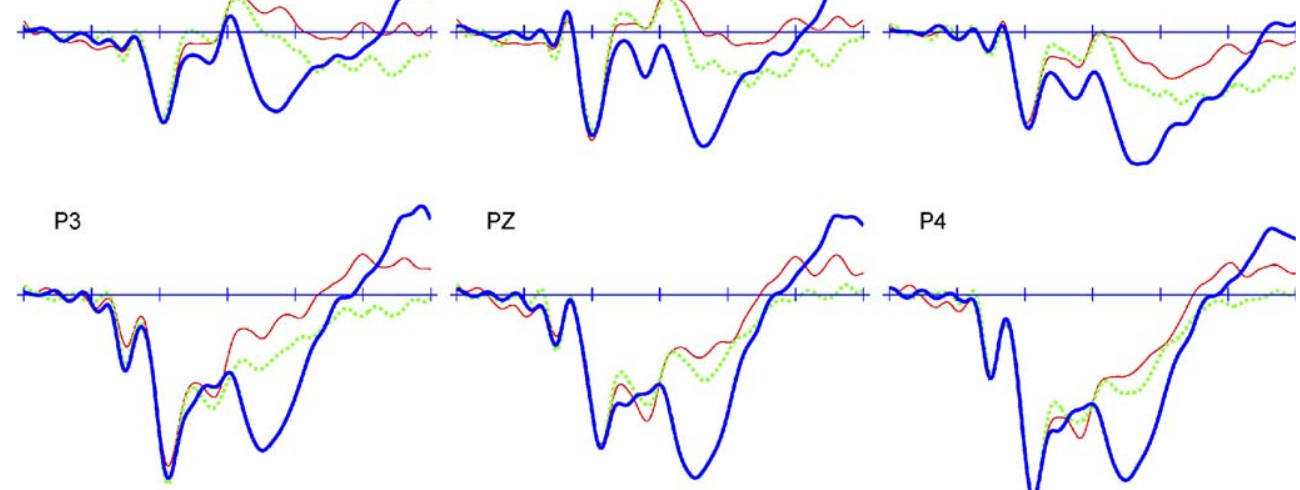

P4
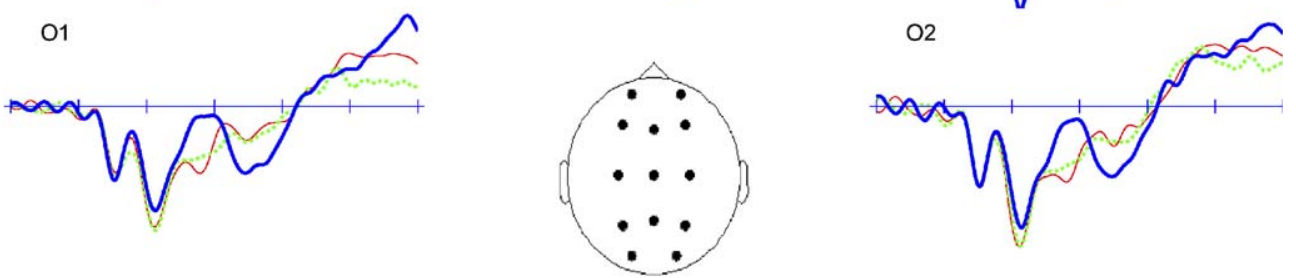

Fig. 3. Waveforms for picture stimuli in the test phase of the Inclusion task. Thin, solid line: new pictures (NP); bold, solid line: old, same-format pictures (PP); dotted line: pictures, studied as words (WP).

In an effort to probe the extension of the Task effect, analyses were performed in each time interval separately ( 2 Tasks $\times 3 \times 3$ electrode sites). There were main effects of Task in the first, $F(1,30)=6.12 ; p=0.019$, and the second intervals, $F(1,30)=5.42 ; p=0.027$, but not in the third. To further elucidate the effects, the analyses were repeated for pictures and for words separately. These results showed main effects of Task in the 500-700 ms range for pictures, $F(1,30)=8.17 ; p=0.008$, and, marginally, in the $300-500 \mathrm{~ms}$ range for words, $F(1,30)=3.92 ; p=0.057$, and pictures, $F(1,30)=3.55 ; p=0.069$.

To test the possibility that the parietal positivity was associated with response confidence rather than retrieval orientation - in view of the fact that the Exclusion group was 

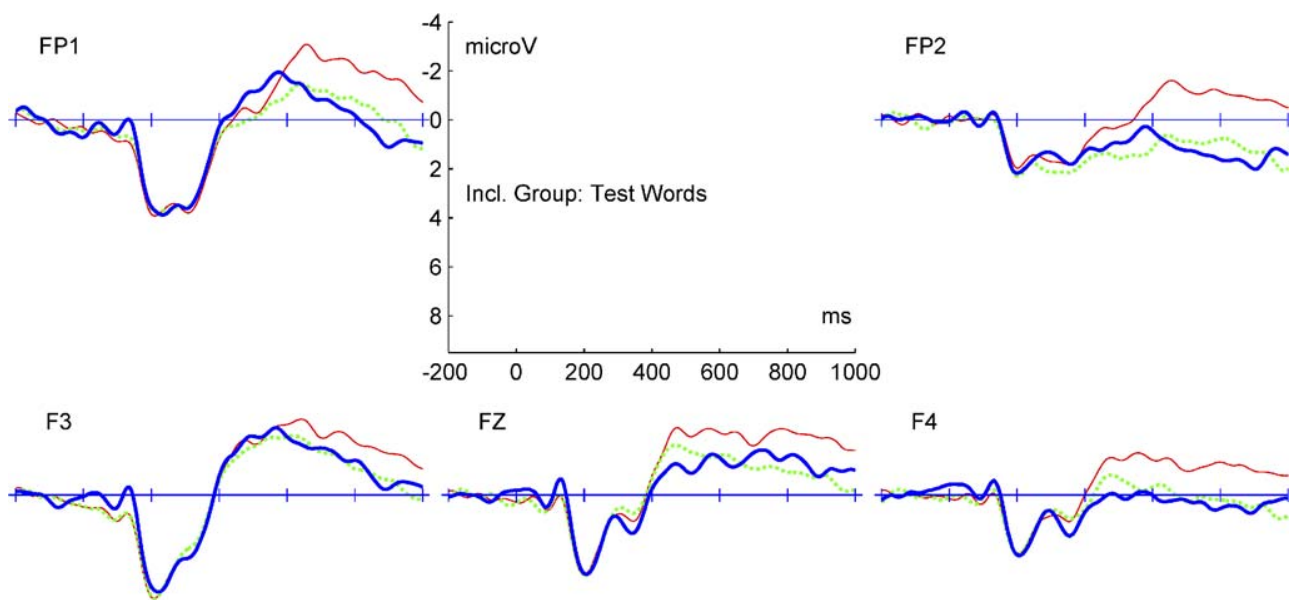

F4

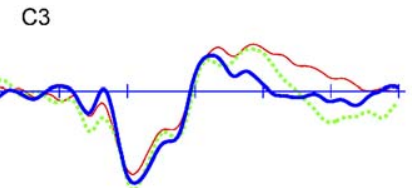

$\mathrm{CZ}$

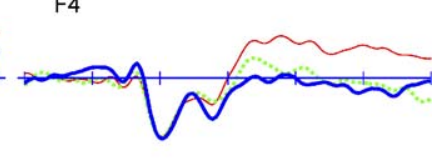

P3

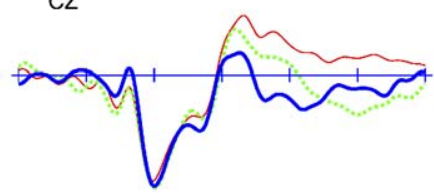

C4

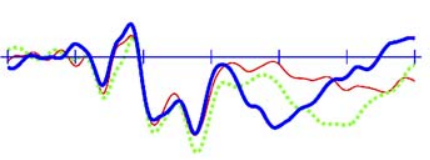

$\mathrm{PZ}$



P4
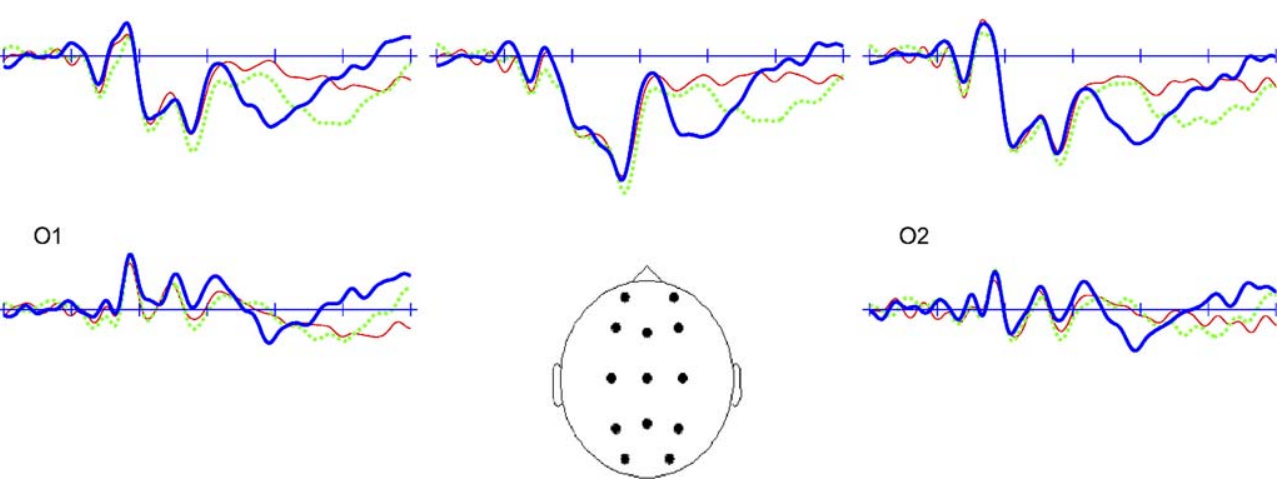

$\mathrm{O} 2$

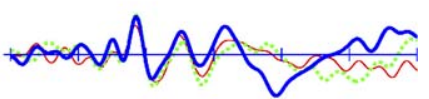

Fig. 4. Waveforms for word stimuli in the test phase of the Inclusion task. Thin, solid line: new words (NW); bold, solid line: old, same-format words (WW); dotted line: words, studied as pictures (PW).

more accurate (hence probably more confident) in the New Picture condition than the Inclusion group - an additional analysis was performed. On the basis of their accuracy in rejecting new distractors, the participants were divided into four groups. This variable (i.e. performance level) was introduced as another factor in the Anova reported in Table 4. If confidence was the causative factor, Performance would explain the variance better than Exclusion/Inclusion Task. In fact, the analysis changed very little. Task still interacted reliably [Task $\times$ Format $\times$ Ant/Post: $F(2,50)=4.18 ; p=0.043$, and follow-up tests still showed a reliable Task effect on parietal amplitudes: $F(1,25)=4.09 ; p=0.05$. The main effect of Task in the main analysis was modified into a Task $\times$ Format interaction, $F(1,25)=4.99$; $p=0.035$. The Performance factor also interacted significantly with Format, $F(3,25)=3.73$; 

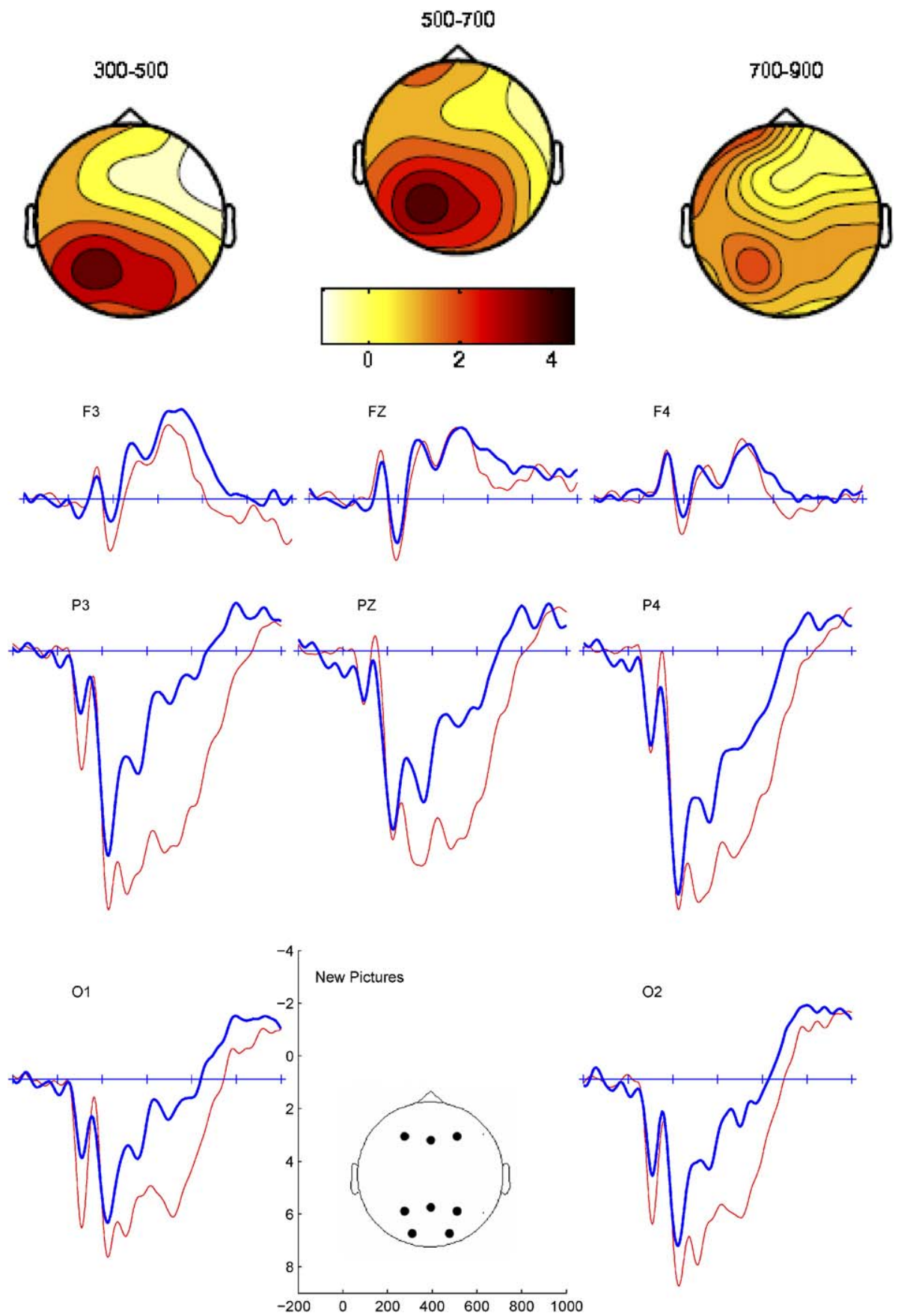

Fig. 5. Correct rejection ERPs for the Exclusion task (thin line) and the Inclusion task (bold line) in the new picture (NP) condition. Electrode positions shown in inset map. The top row of topographical maps shows mean differences between tasks (Exclusion-Inclusion) in three time intervals. 

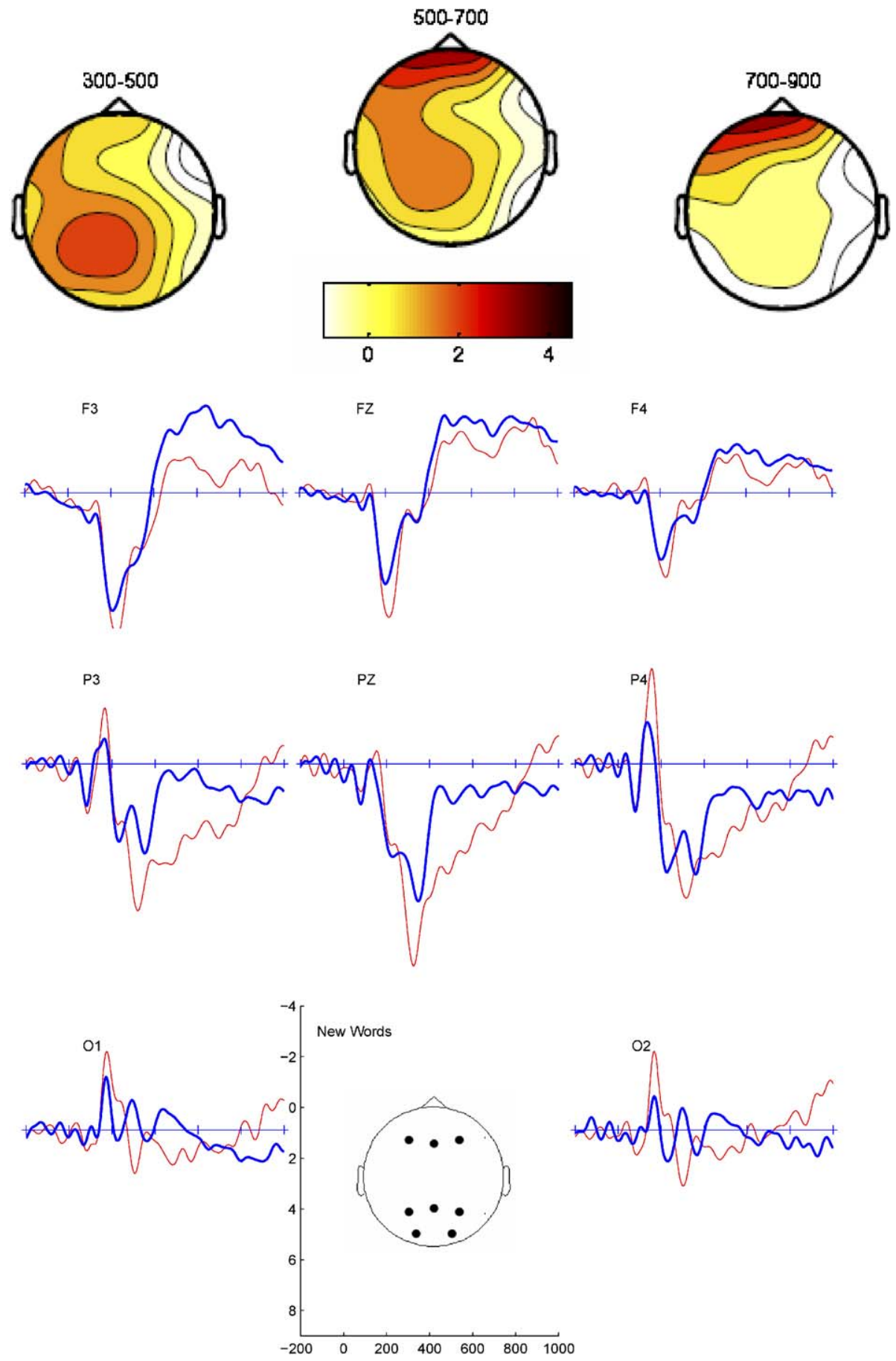

Fig. 6. Correct rejection ERPs for the Exclusion task (thin line) and the Inclusion task (bold line) in the new word (NW) condition. Electrode positions shown in inset map. The top row of topographical maps shows mean differences between tasks (Exclusion-Inclusion) in three time intervals. 
Table 4

Outcome of an overall Anova of correct rejection ERPs

\begin{tabular}{llrrrr}
\hline Source & df1 & df2 & $\varepsilon$ & \multicolumn{2}{c}{$p$} \\
\hline Task & $\mathbf{1}$ & $\mathbf{3 0}$ & $\mathbf{1 . 0 0}$ & $\mathbf{4 . 5 2 0}$ & $\mathbf{0 . 0 4 2}$ \\
Interval & 2 & 60 & 0.88 & 9.043 & 0.001 \\
Format & 1 & 30 & 1.00 & 5.591 & 0.025 \\
Ant-post & 2 & 60 & 0.67 & 32.502 & $<0.001$ \\
Interval $*$ format & 2 & 60 & 0.73 & 5.304 & 0.015 \\
Interval $*$ ant-post & 4 & 120 & 0.41 & 17.145 & $<0.001$ \\
Task $*$ format $*$ ant-post & $\mathbf{2}$ & $\mathbf{6 0}$ & $\mathbf{0 . 6 1}$ & $\mathbf{3 . 9 1 3}$ & $\mathbf{0 . 0 4 8}$ \\
Interval $*$ format $*$ ant-post & 4 & 120 & 0.39 & 38.186 & $<0.001$ \\
Interval $*$ lat & 4 & 120 & 0.69 & 3.412 & 0.024 \\
Interval $*$ format $*$ lat & 4 & 120 & 0.57 & 8.308 & $<0.001$ \\
Ant-post $*$ lat & 4 & 120 & 0.56 & 4.585 & 0.011 \\
Interval $*$ ant-post $*$ lat & 8 & 240 & 0.54 & 5.533 & $<0.001$ \\
Interval $*$ format $*$ ant-post $*$ lat & 8 & 240 & 0.45 & 9.005 & $<0.001$ \\
\hline
\end{tabular}

The analysis, which is mainly aimed at Task (retrieval orientation) effects, covers three time intervals, and includes a $3 \times 3$ central grid of electrodes (F3, Fz, F4, C3, Cz, C4, P3, Pz, P4). Only reliable effects have been included. Effects involving task are shown in bold typeface.

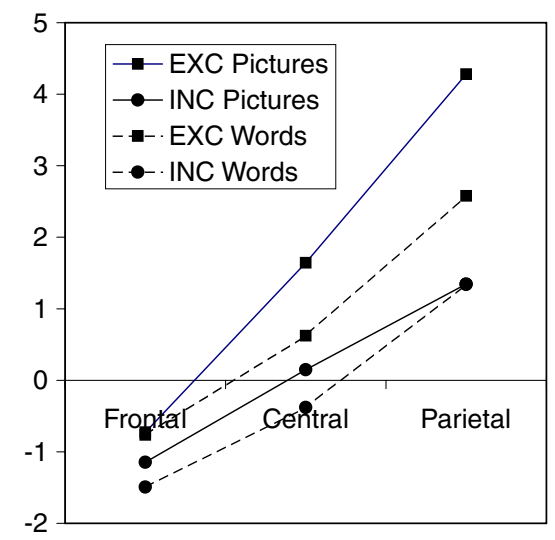

Fig. 7. Mean amplitudes over frontal, central and parietal electrodes for correctly rejected new pictures and words in the Exclusion and Inclusion tasks. Amplitude values have been collapsed across three time intervals: 300-500, 500-700, and 700-900. The Task $\times$ Format $\times$ Site interaction was reliable, due to large posterior amplitudes for pictures in the Exclusion task.

$p=0.024$, but did not otherwise enter into reliable effects, except in one difficult-to-interpret five-way interaction, also including Task.

In summary, correct rejection ERPs showed Task effects over a wide area and for a duration of at least $400 \mathrm{~ms}$. There were Task-related differences for both pictures and words, but they were larger for pictures, and this was especially so over posterior areas. The effect of Task appeared to be independent of performance level.

7.5.2.2. Memory retrieval: old-new effects. To examine successful memory retrieval, we formed old-new differences, i.e. the differences PP-NP, PW-NW, etc. Mean amplitudes of these difference waveforms were analyzed in three time intervals: 300-500, 500-700, and $700-900 \mathrm{~ms}$, with data from a $3 \times 3$ grid of electrodes, covering frontal, central and parietal 
areas. An overview is shown in Table 4, which presents the outcome of Task (Exclusion/ Inclusion $) \times$ Format (Picture/Word) $\times$ Anterior-Posterior location (frontal, central, parietal) $\times$ Lateral position (left, midline, right) analyses. Main effects are presented, and interactions, where significant. The intercept term is a measure of the overall old-new effect, i.e. whether the difference waveform is different from zero (Table 5).

The old-new waveforms from the three midline electrodes are shown in Fig. 8.

7.5.2.3. Same-format items. There were overall old-new (intercept) effects in all three time bands, with a maximum in the middle (500-700) interval. For the same-format items, which were to elicit the same responses in both groups, there were no differences between tasks, nor were there any interactions involving Task.

Format had a differentiating effect, because pictures showed a stronger response in the early interval (300-500), words were stronger in the late (700-900) interval, and there was no difference in the middle band. Format also interacted with location.

Location effects generally indicated a central maximum (at electrode $\mathrm{Cz}$ ) of old-new amplitudes. However, the Format factor significantly modified this pattern, indicating that the retrieval process was served by different brain areas when recognising pictures as compared to when recognising words. This was particularly apparent in the late interval, where word recognition developed into bilateral, frontal activation, whereas picture recognition had a right hemispheric late maximum (Fig. 9).

7.5.2.4. Cross-format items. The cross-format categories showed marked Task effects in all three time bands. In all cases, this indicated more positive old-new differences in the Inclusion task, while Exclusion amplitudes hovered around zero. When Task groups were analysed separately, Inclusion participants had significant old-new (intercept) effects in both the middle and the late intervals $[F(1,15)=11.78 ; p=0.004$, and $F(1,15)=14.25 ; p=0.002]$, but Exclusion participants had no intercept effect in any time band.

To examine whether the scalp distributions of old-new amplitudes were different for cross-format recognition as compared to same-format recognition, an additional analysis was performed in the Inclusion group only (because only this group showed old-new effects to both). The analysis comprised four factors: Study-Test Congruence (same-format, cross-format), Test Format (picture, word), Anterior-Posterior position and Lateral position, and the interest focused on interactions between Congruence and the position factors. In the 500-700 interval, Congruence interacted with the Anterior-Posterior factor, $F(2,30)=6.98 ; \varepsilon=0.58 ; p=0.014$. After rescaling (McCarthy \& Wood, 1985), the effect remained significant, $F(2,30)=5.32 ; \varepsilon=0.63 ; p=0.026$. The interaction was due to an increasing gradient from front to back in the same-format conditions, and from back to front in the cross-format conditions.

7.5.2.5. Onset pattern of old-new effects. Among preserved-format items, pictures (PP) showed an early, frontal effect, lasting from 300 to $600 \mathrm{~ms}$, followed by a parietal effect from 400 to $600 \mathrm{~ms}$. Words (WW) developed somewhat later effects with no clear frontal precedence.

Cross-format items produced old-new effects only in the Inclusion task, and these were characterized by the fact that there was no marked lag between frontal and parietal effects, i.e. no early frontal positivity preceding the later widespread effect. 


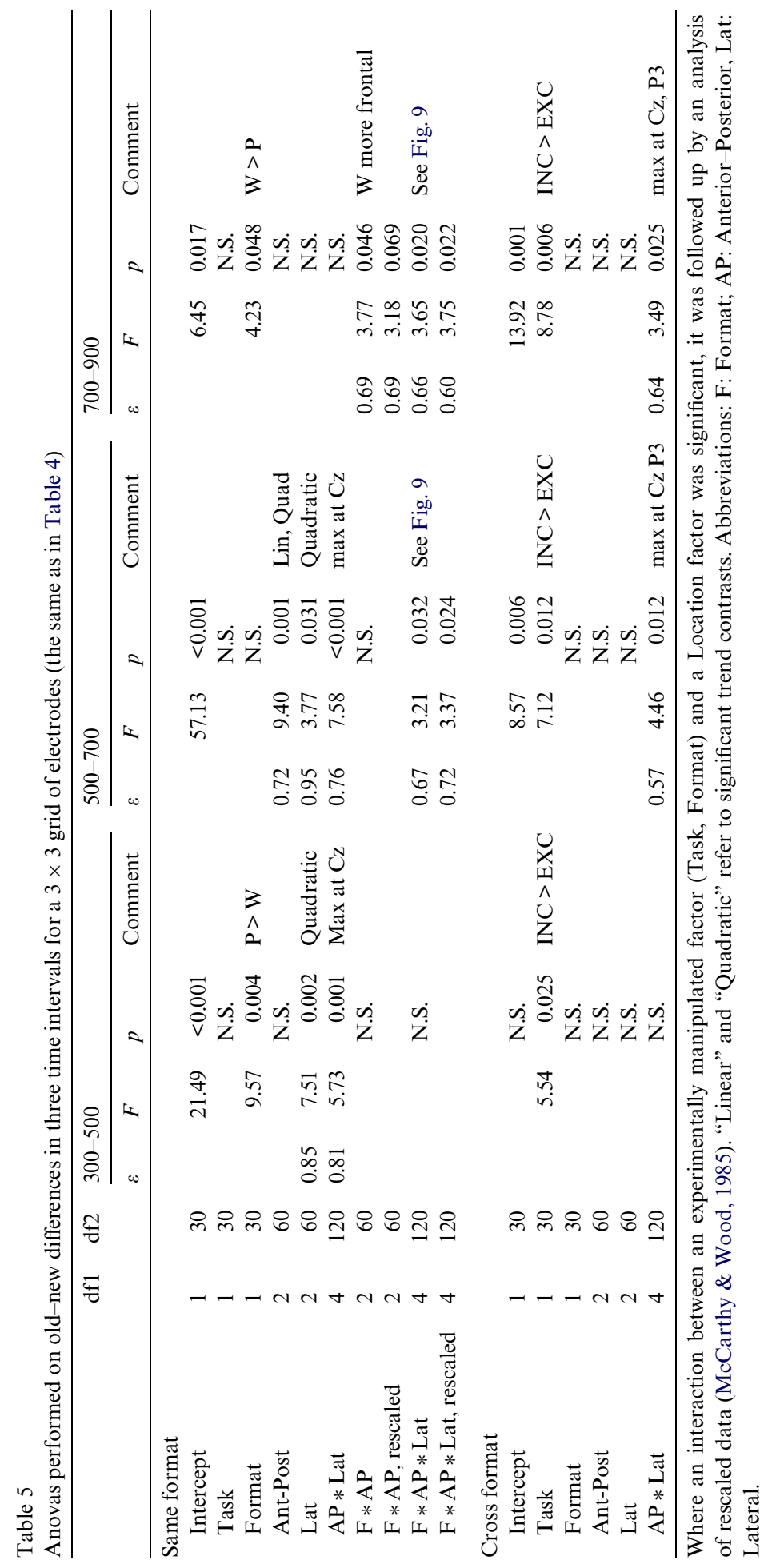




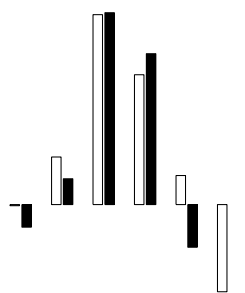

PP $\quad F Z$

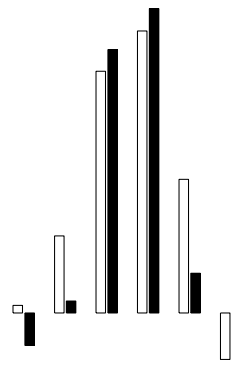

PP $\quad C Z$

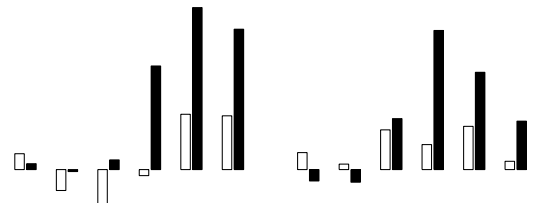

WP FZ



WW FZ



PP PZ

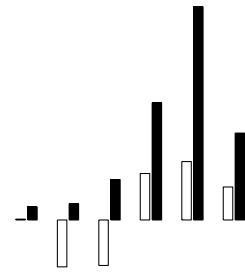

PW CZ



WP $\mathrm{CZ}$

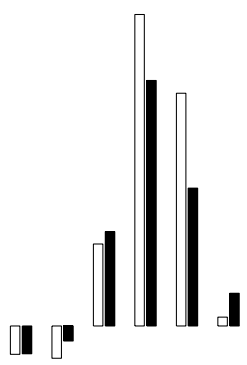

WW CZ

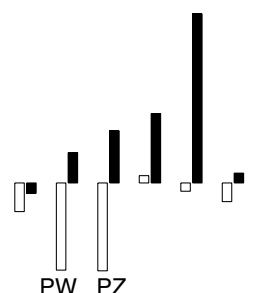

PW PZ

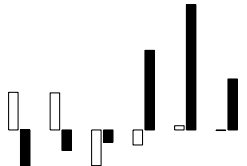

WP PZ
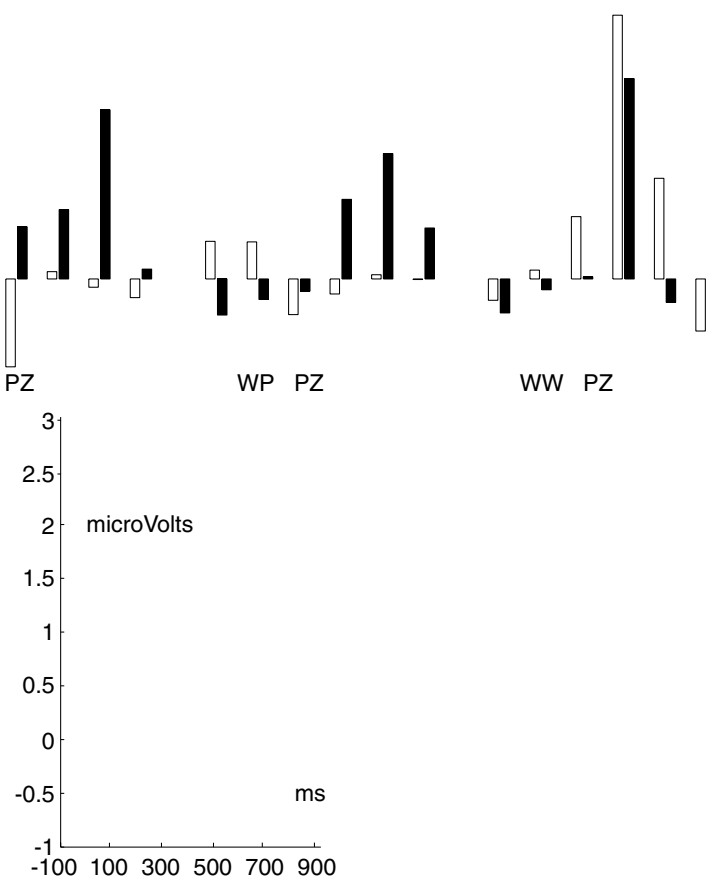

Fig. 8. Old-new differences in the two same-format conditions (PP and WW, left and right) conditions and the two cross-format conditions (PW and WP, middle columns). Dark bars: Inclusion task; light bars: Exclusion task. Electrode positions Fz, Cz and Pz along the midline. Amplitudes have been averaged over successive 200-ms epochs, starting with -100 to 100 and ending with $900-1100$ ms. Note essentially zero old-new effects in the Exclusion task for cross-format items (PW and WP). 
PP both groups
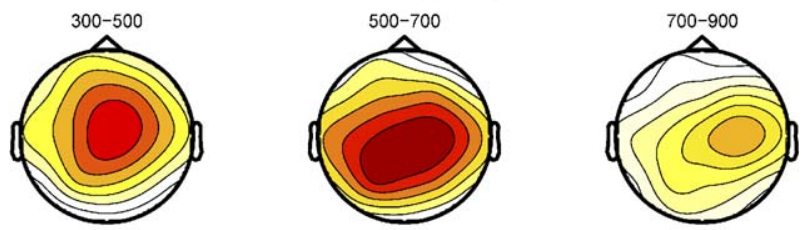

WW both groups
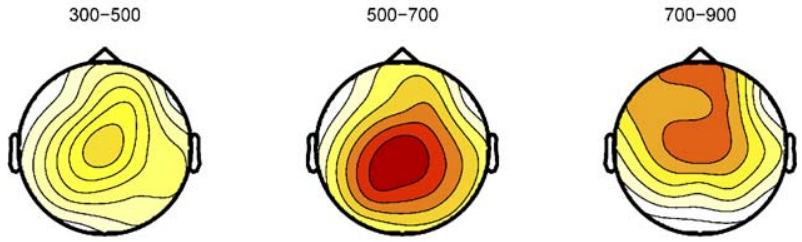

Same-format Inclusion condition
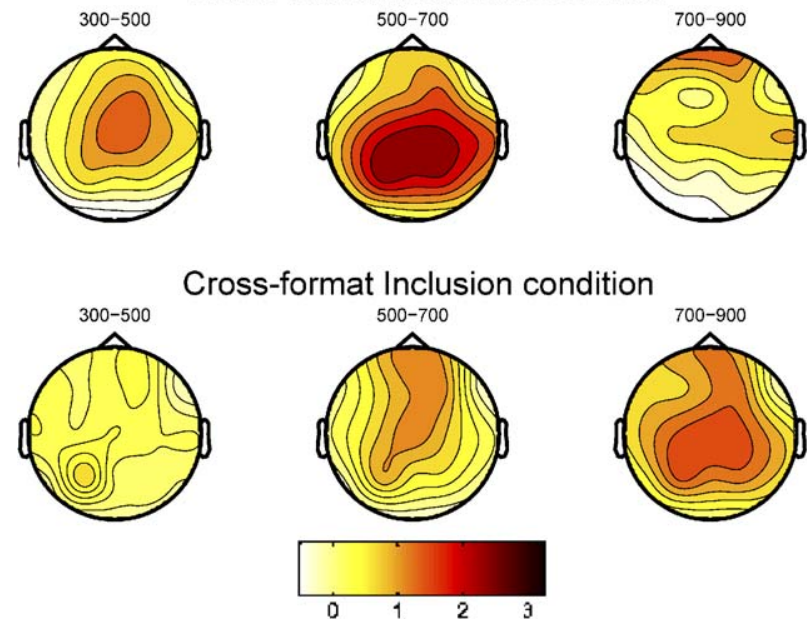

Fig. 9. Scalp distribution of old-new effects for same-format items, averaged over tasks (top two rows). Retrieval patterns for pictures and words show differences from each other, particularly in the late interval, where words evoke a more frontal distribution. In the Inclusion task (lower two rows), the same-format conditions (collapsed over PP and WW) evoke a more posterior distribution than the cross-format conditions (collapsed over PW and WP).

To test these visual impressions, onset latencies of the old-new effect for all four conditions were analyzed in the Inclusion group. (The question was not applicable in the Exclusion group, which showed no old-new effect in the cross-format conditions.)

A 2 (Congruence: same/cross-format) $\times 2$ (Test format: picture/word) $\times 3$ (AnteriorPosterior) $\times 3$ (Lateral position) analysis of onset latencies showed a Congruence main effect, $F(1,15)=18.57 ; p=0.001$, modified by a Congruence $\times$ Test Format interaction, $F(1,15)=6.19 ; p=0.025$. This was due to particularly short latencies in the PP condition. More importantly, there were interactions with the Anterior-Posterior factor, pertinent to the question whether frontal effects precede parietal ones. The Congruence $\times$ Ant $/$ Post interaction was reliable, $F(2,60)=5.36 ; \varepsilon=0.74 ; p=0.019$, as was the Congruence $\times$ 
Table 6

Onset latencies of old-new effects in the Inclusion group, calculated as described in the Methods section

\begin{tabular}{lllll}
\hline & PP & WW & WP & PW \\
\hline Frontal & 248 & 330 & 342 & 410 \\
Central & 284 & 345 & 379 & 307 \\
Parietal & 310 & 360 & 350 & 344 \\
\hline
\end{tabular}

Averages have been collapsed across the Lateral factor (left-centre-right).

Format $\times$ Ant $/$ Post interaction, $F(2,60)=5.47 ; \varepsilon=0.87 ; p=0.013$. Mean onset latencies are presented in Table 6 .

To parse the interaction, latencies were analyzed for each condition separately, with a view to determining whether the linear trend contrast over the Anterior-Posterior factor was significant, thus indicating a frontal $<$ central $<$ parietal ordering of onsets. This contrast was significant only for the PP condition, $F(1,15)=7.75 ; p=0.014$, whereas all other $F \mathrm{~s} \leqslant 3.11 ;$ N.S.

\subsection{Discussion}

\subsubsection{The mirror effect}

Using a format exclusion/inclusion task, we induced a perceptual and a conceptual retrieval orientation. This manipulation dissociated the high end from the low end of the picture-word mirror effect. The rejection part of the mirror effect could be manipulated independently of the hit-rate part, and it seemed to have a specific physiological signature, arguably resulting from confrontation with unfamiliar perceptual features, leading to a swift "New" decision.

Johns and Mewhort have recently argued that positive and negative recognition decisions may be based on partly different sources of evidence:" We believe that subjects assess both familiarity and evidence for contradiction. Sufficient familiarity information will prompt a yes response; sufficient contradictory information will prompt a no response. Although the two kinds of evidence will be correlated, they need not be perfectly correlated, and a single-criterion decision model does not apply" (Johns \& Mewhort, 2003, p. 286). The present data give support to the notion that positive and negative decisions can rest on different types of informations. For positive decisions on same-format test items, both perceptual and conceptual clues were available in both tasks. For new items, the available information differed as a function of format and task, in the following way.

The rejection of new pictures was easier than the rejection of new words, if a perceptual orientation was adopted, but not with a conceptual orientation. Arguably, unfamiliar visual features of pictures stand out and facilitate rejection, more so than is the case for unfamiliar words. An unfamiliar picture often has perceptual features with low prior probabilities of occurrence - an unexpected colour or texture that readily flags the picture as unseen. The present findings are in keeping with a frequency-judgement study (Hintzman, Curran, \& Caulton, 1995) which found that familiarity values of pictures started lower than those of words but increased more rapidly with repetition. If perceptual features are disregarded, this advantage is lost.

Although older memory models had difficulties explaining the presence of the mirror effect, more recent models (Glanzer et al., 1993; Shiffrin \& Steyvers, 1997) incorporate a 
mechanism - the likelihood ratio scale - that derives the mirror effect as a natural consequence. However, this capacity comes at a cost. The mechanism is a rigid one, in that it does not allow exceptions from the rule. Wherever hit rates for a stimulus type is higher, its false alarm rate must be lower - the two move in tandem (Hilford, Glanzer, \& Kim, 1997). Therefore, the present results, where a mirror effect was produced in one condition and eliminated in another condition of the same experiment, seem incompatible with current single-process, single-store models.

\subsubsection{Retrieval orientations}

On the basis of previous investigations (Herron \& Rugg, 2003a; Hornberger et al., 2004; Robb \& Rugg, 2002), we expected a conceptual retrieval orientation to give rise to relatively more negative-going amplitudes than a perceptual orientation. This proved to be the case. The difference between orientations can be described as a negative-going voltage modulation accompanying a conceptual orientation, or a positive-going modulation accompanying a perceptual orientation. It may seem a moot point which alternative is chosen, but one observation favours the alternative in which perceptual orientation is seen as the active agent. The difference between tasks interacted with formats, such that it was differently distributed for new pictures than for new words. This suggests that the perceptual orientation is driving the difference.

The scalp distribution of the task difference had a more posterior distribution for pictures than for words. Some earlier studies of similar orientation differences have found a widespread distribution with a central maximum (Herron \& Rugg, 2003a; Robb \& Rugg, 2002), but the only study to date that used both picture and words as test items (Hornberger et al., 2004) found a similar interaction, with the orientation effect having a more anterior distribution for words than for pictures.

The format-specific pattern suggests to us that perceptual feature extraction may be the driving force behind the orientation effect, but other interpretations are perhaps possible. A candidate cause may be difficulty level, or, relatedly, response confidence, both of which could influence the late positive complex (LPC). The LPC has a broadly posterior distribution, and covaries in size with task relevance, stimulus probability (inversely), and response confidence. Robb and Rugg (2002) examined task difficulty, and with it presumably confidence, as a separate manipulation, but found it to be unrelated to the orientation effect. Also, Curran (2004) found response confidence to influence amplitude for old, but not for new, stimuli in a recognition task. Rubin, Van Petten, Glisky, and Newberg (1999) also examined confidence effects and found a similar pattern, with clear confidence effects on ERPs for old items, but only a non-significant tendency for new items $(p<.10)$. It is also worth noting that this tendency, weak though it was, was found in a relatively late interval (600-900), as is the case for other response confidence effects. Our task effect, on the other hand, had an early onset $(300 \mathrm{~ms})$, and was terminated at $700 \mathrm{~ms}$, clearly separated from response execution, which had an average latency of at least $1000 \mathrm{~ms}$.

The format-specific scalp distribution in our data also suggests that the orientation effect cannot be reduced to a generic factor, such as response confidence. Stimulus probability, another known LPC influence, also seems unlikely as a cause of the orientation effect, because to-be-rejected stimuli were more frequent in the Exclusion task, where positive amplitudes were large, i.e. the reverse of a stimulus probability effect.

In the present results, measures of performance - both accuracy and reaction time were equal across tasks for new words, which implies that the efforts invested by the two 
groups were equivalent, at least for this type of stimuli. Still, correct rejections of words showed a task effect, which can therefore be ascribed to retrieval orientation, rather than retrieval effort. Furthermore, a supplementary analysis showed that Exclusion/Inclusion task effects were not reducible to the influence of performance accuracy (or, indirectly, response confidence).

\subsubsection{Old-new effects}

The findings of the present study have many similarities with those of Schloerscheidt and Rugg (2004), who studied the picture-word format-change paradigm with instructions resembling those of the present Inclusion task. Extending earlier research (Kazmerski \& Friedman, 1997; Schloerscheidt \& Rugg, 2004), we had an Exclusion condition, with the purpose of testing whether involuntary, conceptual memory was detectable in the ERPs. We found no evidence of any old-new effect whatsoever with cross-format items in the Exclusion task. This is in some contrast with the reaction time results in both our experiments, which showed a slowing-down of the "New" responses to cross-format items, relative to completely unacquainted items. Thus, there were behavioural signs of some conceptually based recognition, perhaps a sense of familiarity that, although irrelevant for the task given to the Exclusion participants, affected their decision latency. This process, reproduced behaviourally in both experiments, did not leave any marks on the ERPs.

Other studies have found cross-modal effects in the latency range of the FN400 (Domalski, Smith, \& Halgren, 1991; Holcomb, Anderson, \& Grainger, 2005) when the task did not involve explicit recognition. These studies have relied on verbal stimuli that appeared in auditory and visual formats. The two representations are separated by sensory modalities, yet can be expected to share some (possibly phonological) representation, more so than pictures and words, which share representations only at an abstract, semantic level. More notably still, there have been findings (Dzulkifli \& Wilding, 2005; Herron \& Wilding, 2005) of early, frontal old-new effects even for stimuli that were explicitly excluded by instructions. In these studies, recognition was tested by visual words that had been studied in either of two orienting tasks, one of which was designated as the target. In this context, non-target stimuli produced an early (300-500) frontal, but no parietal, old-new effect. For the interpretation of this effect it is important to note that test probes were exact perceptual copies of the studied exemplars, and the results are therefore compatible with the view that the early familiarity effect is linked to perceptual processes.

Our results are roughly consistent with a perceptually specific interpretation of the early familiarity effect. However, we would not wish to carry this interpretation too far on the basis of our own data, because we found an FN400 effect only for the picture-picture condition, not for the word-word condition. The latter has often produced FN400 effects in earlier investigations, and without specific information as to why we did not find the wordword effect, we do not wish to base far-reaching conclusions on the absence of the effect for cross-format repetitions.

Turning to the parietal positivity, the absence of recollection effects in the ERPs to excluded, although in some sense recognised, items supports the observation of Herron and Rugg (2003b) that recollection is not a necessary condition for an exclusion decision. Instead, the data suggest that negative decisions can be based on a subset of the available evidence. Other recent studies have corroborated the case for strategic influences on 
recollection (Dzulkifli \& Wilding, 2005; Herron \& Wilding, 2005) by showing that recollection can be short-circuited out of the path to a correct response, if other evidence is available and sufficient. In our study, non-matching perceptual features could fast-track a rejection response, in the process overriding the matching conceptual features that remained unheeded in their call for further retrieval.

Interestingly, old-new effects varied significantly in their scalp distribution with the type of material to be retrieved. First, changed-format items exhibited more frontal old-new differences than same-format items in the 500-700 ms range. Arguably, the more demanding task of recognition across a format change may engage the frontal lobes, more so than same-format recognition. Also, within the category of same-format recognition, studied words produced more frontally distributed old-new differences in the 700-900 ms band than did pictures, possibly because the more demanding word recognition engages the frontal lobes, or because picture recognition can rely on perceptual qualities to a higher degree.

\subsubsection{Conclusions}

As a whole, the present data are compatible with views that emphasize the malleability and diversity of retrieval processing, such as two-process theories of recognition memory (Mandler, 1980; Yonelinas, 2002). Behaviourally, the results of both experiments show that the rejection part of the mirror effect can be dissociated from the hit-rate part. By this token, at least two processes seem to be involved, for a single strength continuum is not sufficient to explain the reversal of the mirror effect between the two task conditions (Reder et al., 2000). Electrophysiologically, the evidence indicated that memory processing - to the extent that it was discernible in scalp-recorded ERPs - was voluntarily controlled, for there was no vestige of old-new effects in the Exclusion group's cross-format responses. Overall, ERPs gave a strong impression of the degree to which negative perceptual clues can preclude further contact with the memory trace. In contrast, reaction times suggested some involuntary effects of cross-format recognition, possibly pertaining to hesitancy in the decision stage. Alternatively, it could indicate memory processing simply not detectable in ERPs.

Changes in scalp distribution of old-new differences in ERPs suggested that different neuronal populations were active in retrieval depending on the material accessed - more anterior areas tended to be recruited in conceptually demanding retrieval, and more posterior areas in perceptually reliant retrieval.

The analysis of ERPs to correctly rejected foils revealed effects of retrieval orientation. Memory retrieval can be directed toward specific subsets of memory by cognitive sets, induced by task demands. In particular, perceptual and conceptual features form such subsets. With a perceptual retrieval orientation, quick negative decisions are facilitated by selective attention to the perceptual features of the cue. This process is accompanied by large positive amplitudes in event-related brain potentials.

\section{Acknowledgements}

This study was financed by grants from the Swedish Research Council to Georg Stenberg and Ingmar Rosén (\#84 and 421-2002-3003). We gratefully acknowledge assistance in data collection from Sara Denward for Experiment 1 and Bodil Persson for Experiment 2. 


\section{References}

Aggleton, J. P., \& Brown, M. W. (1999). Episodic memory, amnesia, and the hippocampal-anterior thalamic axis. Behavioral and Brain Sciences, 22(3), 425-489.

Allan, K., \& Rugg, M. D. (1997). An event-related potential study of explicit memory on tests of cued recall and recognition. Neuropsychologia, 35(4), 387-397.

Arndt, J., \& Reder, L. M. (2002). Word frequency and receiver operating characteristic curves in recognition memory: evidence for a dual-process interpretation. Journal of Experimental Psychology: Learning, Memory, and Cognition, 28(5), 830-842.

Brown, M. W., \& Aggleton, J. P. (2001). Recognition memory: what are the roles of the perirhinal cortex and hippocampus? Nature Reviews Neuroscience, 2(1), 51-61.

Clark, S. E., \& Gronlund, S. D. (1996). Global matching models of recognition memory: how the models match the data. Psychonomic Bulletin and Review, 3(1), 37-60.

Curran, T. (2000). Brain potentials of recollection and familiarity. Memory and Cognition, 28(6), 923-938.

Curran, T. (2004). Effects of attention and confidence on the hypothesized ERP correlates of recollection and familiarity. Neuropsychologia, 42(8), 1088-1106.

Curran, T., \& Cleary, A. M. (2003). Using ERPs to dissociate recollection from familiarity in picture recognition. Cognitive Brain Research, 15(2), 191-205.

Curran, T., \& Dien, J. (2003). Differentiating amodal familiarity from modality-specific memory processes: an ERP study. Psychophysiology, 40(6), 979-988.

Domalski, P., Smith, M. E., \& Halgren, E. (1991). Cross-modal repetition effects on the N4. Psychological Science, 2(3), 173-178.

Düzel, E., Yonelinas, A. P., Mangun, G. R., Heinze, H.-J., \& Tulving, E. (1997). Event-related brain potential correlates of two states of conscious awareness in memory. Proceedings of the National Academy of Sciences, 94 , 5973-5978.

Dzulkifli, M. A., \& Wilding, E. L. (2005). Electrophysiological indices of strategic episodic retrieval processing. Neuropsychologia, 43(8), 1152-1162.

Finnigan, S., Humphreys, M. S., Dennis, S., \& Geffen, G. (2002). ERP ‘old/new’ effects: memory strength and decisional factor(s). Neuropsychologia, 40(13), 2288-2304.

Glanzer, M., \& Adams, J. K. (1985). The mirror effect in recognition memory. Memory and Cognition, 13(1), 8-20.

Glanzer, M., Adams, J. K., Iverson, G. J., \& Kim, K. (1993). The regularities of recognition memory. Psychological Review, 100(3), 546-567.

Herron, J. E., \& Rugg, M. D. (2003a). Retrieval orientation and the control of recollection. Journal of Cognitive Neuroscience, 15(6), 843-854.

Herron, J. E., \& Rugg, M. D. (2003b). Strategic influences on recollection in the exclusion task: electrophysiological evidence. Psychonomic Bulletin and Review, 10(3), 703-710.

Herron, J. E., \& Wilding, E. L. (2005). An electrophysiological investigation of factors facilitating strategic recollection. Journal of Cognitive Neuroscience, 17(5), 777-787.

Hilford, A., Glanzer, M., \& Kim, K. (1997). Encoding, repetition, and the mirror effect in recognition memory: symmetry in motion. Memory and Cognition, 25(5), 593-605.

Hintzman, D. L., Curran, T., \& Caulton, D. A. (1995). Scaling the episodic familarities of pictures and words. Psychological Science, 6(5), 308-313.

Holcomb, P. J., Anderson, J. M., \& Grainger, J. (2005). An electrophysiological study of cross-modal repetition priming. Psychophysiology, 42, 493-507.

Hornberger, M., Morcom, A. M., \& Rugg, M. D. (2004). Neural correlates of retrieval orientation: effects of studytest similarity. Journal of Cognitive Neuroscience, 16(7), 1196-1210.

Jacoby, L. L. (1991). A process dissociation framework - separating automatic from intentional uses of memory. Journal of Memory and Language, 30(5), 513-541.

Johns, E. E., \& Mewhort, D. J. K. (2003). The effect of feature frequency on short-term recognition memory. Memory and Cognition, 31(2), 285-296.

Joordens, S., \& Hockley, W. E. (2000). Recollection and familiarity through the looking glass: when old does not mirror new. Journal of Experimental Psychology: Learning, Memory, and Cognition, 26(6), 1534-1555.

Kazmerski, V. A., \& Friedman, D. (1997). Old/new differences in direct and indirect memory tests using pictures and words in within- and cross-form conditions: event-related potential and behavioral measures. Cognitive Brain Research, 5(4), 255-272.

Mandler, G. (1980). Recognizing: the judgment of previous occurrence. Psychological Review, 87(3), $252-271$. 
McCarthy, G., \& Wood, C. C. (1985). Scalp distributions of event-related potentials: an ambiguity associated with analysis of variance models. Electroencephalography and Clinical Neurophysiology, 62(3), 203-208.

Mecklinger, A. (2000). Interfacing mind and brain: a neurocognitive model of recognition memory. Psychophysiology, 37(5), 565-582.

Mintzer, M. Z., \& Snodgrass, J. G. (1999). The picture superiority effect: support for the distinctiveness model. American Journal of Psychology, 112(1), 113-146.

Nessler, D., Mecklinger, A., \& Penney, T. B. (2001). Event related brain potentials and illusory memories: the effects of differential encoding. Cognitive Brain Research, 10(3), 283-301.

Olichney, J. M., Van Petten, C., Paller, K. A., Salmon, D. P., Iragui, V. J., \& Kutas, M. (2000). Word repetition in amnesia: electrophysiological measures of impaired and spared memory. Brain, 123(9), 1948-1963.

Paller, K. A. (2004). Electrical signals of memory and of the awareness of remembering. Current Directions in Psychological Science, 13(2), 49-55.

Paller, K. A., \& Kutas, M. (1992). Brain potentials during memory retrieval provide neurophysiological support for the distinction between conscious recollection and priming. Journal of Cognitive Neuroscience, 4(4), $375-391$.

Ranganath, C., \& Paller, K. A. (2000). Neural correlates of memory retrieval and evaluation. Cognitive Brain Research, 9(2), 209-222.

Ratcliff, R., \& McKoon, G. (2000). Memory models. In E. Tulving \& F. Craik (Eds.), Oxford handbook of memory. Oxford: Oxford University Press.

Reder, L. M., Angstadt, P., Gary, M., Erickson, M. A., \& Ayers, M. S. (2002). A reexamination of stimulus-frequency effects in recognition: two mirrors for low- and high-frequency pseudowords. Journal of Experimental Psychology: Learning, Memory, and Cognition, 28(1), 138-152.

Reder, L. M., Nhouyvanisvong, A., Schunn, C. D., Ayers, M. S., Angstadt, P., \& Hiraki, K. (2000). A mechanistic account of the mirror effect for word frequency: a computational model of remember-know judgments in a continuous recognition paradigm. Journal of Experimental Psychology: Learning, Memory, and Cognition, 26(2), 294-320.

Robb, W. G. K., \& Rugg, M. D. (2002). Electrophysiological dissociation of retrieval orientation and retrieval effort. Psychonomic Bulletin and Review, 9(3), 583-589.

Rubin, S. R., Van Petten, C., Glisky, E. L., \& Newberg, W. M. (1999). Memory conjunction errors in younger and older adults: event-related potential and neuropsychological data. Cognitive Neuropsychology, 16(3-5), 459488.

Rugg, M., \& Allan, K. (2000). Event-related potential studies of memoryThe oxford handbook of memory Oxford: Oxford University Press (pp. 521-537).

Rugg, M. D., Allan, K., \& Birch, C. S. (2000). Electrophysiological evidence for the modulation of retrieval orientation by depth of study processing. Journal of Cognitive Neuroscience, 12(4), 664-678.

Rugg, M. D., \& Wilding, E. L. (2000). Retrieval processing and episodic memory. Trends in Cognitive Sciences, 4(3), 108-115.

Rugg, M. D., \& Yonelinas, A. P. (2003). Human recognition memory: a cognitive neuroscience perspective. Trends in Cognitive Sciences, 7(7), 313-319.

Rösler, F., Heil, M., \& Hennighausen, E. (1995). Exploring memory functions by means of brain electrical topography: a review. Brain Topography, 7(4), 301-313.

Schacter, D. L., Wagner, A. D., \& Buckner, R. L. (2000). Memory systems of 1999. In E. Tulving \& F. Craik (Eds.), Oxford handbook of memory. Oxford: Oxford University Press.

Schloerscheidt, A. M., \& Rugg, M. D. (2004). The impact of change in stimulus format on the electrophysiological indices of recognition. Neuropsychologia, 42(4), 451-466.

Senkfor, A. J., \& Van Petten, C. (1998). Who said what? An event-related potential investigation of source and item memory. Journal of Experimental Psychology: Learning, Memory, and Cognition, 24(4), 1005-1025.

Shiffrin, R. M., \& Steyvers, M. (1997). A model for recognition memory: REM - retrieving effectively from memory. Psychonomic Bulletin and Review, 4(2), 145-166.

Stenberg, G., Radeborg, K., \& Hedman, L. R. (1995). The picture superiority effect in a cross-modality recognition task. Memory and Cognition, 23(4), 425-441.

Van Petten, C., Luka, B. J., Rubin, S. R., \& Ryan, J. P. (2002). Frontal brain activity predicts individual performance in an associative memory exclusion test. Cerebral Cortex, 12(11), 1180-1192.

Wagner, A. D., \& Gabrieli, J. D. E. (1998). On the relationship between recognition familiarity and perceptual fluency: evidence for distinct mnemonic processes. Acta Psychologica, 98(2-3), 211-230.

Weldon, M. S., \& Roediger, H. L. (1987). Altering retrieval demands reverses the picture superiority effect. Memory and Cognition, 15(4), 269-280. 
Weldon, M. S., Roediger, H. L., Beitel, D. A., \& Johnston, T. R. (1995). Perceptual and conceptual processes in implicit and explicit tests with picture fragment and word fragment cues. Journal of Memory and Language, 34(2), 268-285.

Yonelinas, A. P. (2002). The nature of recollection and familiarity: a review of 30 years of research. Journal of Memory and Language, 46(3), 441-517.

Yovel, G., \& Paller, K. A. (2004). The neural basis of the butcher-on-the-bus phenomenon: when a face seems familiar but is not remembered. Neuroimage, 21(2), 789-800. 